\title{
Systematic studies on the effect of linear lattice optics for space-charge limited beams
}

\author{
M. Fitterer" \\ CERN, 1211 Geneva, Switzerland and KIT, 76131 Karlsruhe, Germany \\ C. Carli \\ CERN, 1211 Geneva, Switzerland
}

A. Molodozhentsev

KEK, 305-0801 Tsukuba, Japan

A.-S. Müller

KIT, 76131 Karlsruhe, Germany

(Received 27 March 2015; published 8 December 2015)

\begin{abstract}
The HL-LHC (High Luminosity LHC) project aims to an increase of the luminosity of the LHC by a factor of 10. In order to realize this ambitious goal, the LHC itself has to undergo a major upgrade accompanied by an extensive upgrade of the complete injector complex referred to as LHC injector upgrade (LIU). In the framework of the LIU project, a new rapid cycling synchrotron (RCS) as an alternative to the energy upgrade of the existing PS Booster has been proposed. Motivated by the optics studies conducted for this RCS, the more general question of the influence of the linear optics on the machine performance has been raised. In this paper, we want to investigate this question by comparing different lattices with the final aim of identifying lattice characteristics advantageous under strong space-charge effects.
\end{abstract}

DOI: 10.1103/PhysRevSTAB.18.124201

PACS numbers: 29.20.D-

\section{INTRODUCTION}

One of the main performance limitations of the CERN Proton Synchrotron (PS) Booster are space-charge effects. One of the simplest measures to mitigate space-charge effects is to raise the beam energy. Following this rule an upgrade of the existing PS Booster to a higher injection energy from currently 50 to $160 \mathrm{MeV}$ is planned [1-3] in the framework of the LHC injector upgrade (LIU) [4] in support of the HLLHC project [5]. As an alternative to this rather extensive upgrade of the PS Booster, the construction of a rapid cycling synchrotron (RCS) has been studied at CERN $[6,7]$.

At the time of the design of the PS Booster, the linear optics of the machine had been optimized respecting certain lattice characteristics believed back then to be advantageous under strong space-charge effects. Explicitly, the PS Booster features a very smooth variation of the beam size, a high symmetry and high periodicity and a moderate phase advance per cell. In contrast, the lattice and optics proposed for the RCS in general favor FODO (F: focusing quadrupole, $\mathrm{D}$ : defocusing quadrupole, O: drift space) lattices as the required focusing strength is smaller, and in consequence

\footnotetext{
*Miriam.Fitterer@cern.ch

Published by the American Physical Society under the terms of the Creative Commons Attribution 3.0 License. Further distribution of this work must maintain attribution to the author $(s)$ and the published article's title, journal citation, and DOI.
}

also the required space requirements [6]. Furthermore, a threefold or fourfold symmetry was chosen as three to four dispersion-free sections are required for injection, extraction, $\mathrm{rf}$ and, possibly, collimation. This choice is typical for lattices and optics considered for modern high power hadron machines as, for example, [8-10].

In summary, the main differences between the PS Booster and the RCS optics can be expressed by the following characteristics: (i) variation of the beam size, (ii) symmetry of the lattice and optics, (iii) phase advance per cell, and (iv) inserted straight sections, connected to dispersion suppressor schemes, and in general the influence of dispersion.

Based on the considerations for the PS Booster, it was initially expected that a small variation of the beam size, a high lattice symmetry, a small phase advance per cell, and no inserted straight section would lead to the best performance, but the importance of the individual characteristics was not evident.

This paper aims at studying in detail the importance of the individual characteristics by comparing different lattices and optics with regard to the above-mentioned assumptions. The paper starts with a short introduction of the PS Booster and RCS lattices in Sec. II, followed by a short summary of the beam parameters, the simulation method and the analysis tools used (Sec. III). In Sec. IV the studies of the different lattices and optics are presented addressing the influence of fringe fields (Sec. IVA), of the variation of the beam size (Sec. IV B), of systematic resonances (Secs. IV C and IV D), 
of the phase advance per cell (Sec. IV E) and of the impact of different dispersion suppressor schemes (Sec. IV F). In conclusion, Sec. V summarizes the lattice and optics characteristics which the presented studies indicate to be advantageous in case of space-charge dominated machines.

\section{PS BOOSTER AND RCS LATTICE AND OPTICS}

In this section, a short overview of the PS Booster and RCS lattices and optics is given. In particular, also the differences considered relevant in the presence of spacecharge effects are highlighted.

\section{A. PS Booster optics}

The PS Booster lattice consists of 16 equal triplet cells with the defocusing quadrupole in the center. Two bending magnets are installed on both sides close to the triplet followed by a longer straight section. The working point at injection in the case of high brightness beams is set to $Q_{x}=4.28, Q_{y}=4.55$ and is slowly changed during the ramp from $160 \mathrm{MeV}$ at injection to $2 \mathrm{GeV}$ at extraction. To minimize the emittance growth during the ramp, trim quadrupoles are used for a compensation of the $2 Q_{y}=9$ resonance. The optics at injection for the PS Booster are shown in Fig. 1.

As a consequence of the high periodicity, the lattice does not contain any dispersion-free straight sections and the $\mathrm{H}^{-}$ beam delivered from LINAC4 is injected in the bend-free region between two triplets. The resulting tight requirements and the nonzero dispersion make the injection region the main challenge of the PS Booster upgrade. For the same reason, the rf cavities are also installed between two bends of the triplet cells. As a rf system, the PS Booster uses a double harmonic rf system which allows for a larger bunching factor and therefore weaker space-charge effect. Both cavities will be operated in antiphase with a rf voltage of $8 \mathrm{kV}$ for the $h=1$ cavity and $6 \mathrm{kV}$ for the $h=2$ cavity. The main machine parameters after the upgrade are summarized in Table I.

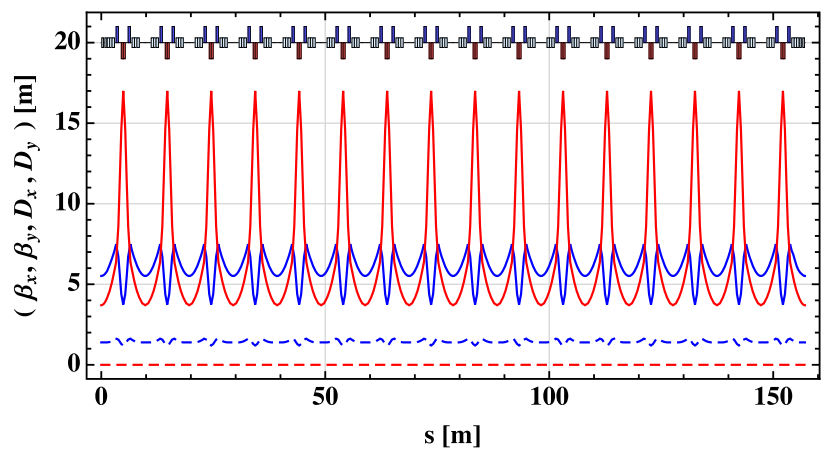

FIG. 1. PS Booster lattice and optics at injection. The $\beta$ function is indicated with a solid line and the dispersion with a dashed line. The horizontal plane is shown in blue and the vertical plane in red.
TABLE I. PS Booster parameters after the upgrade.

\begin{tabular}{lc}
\hline \hline Injection kinetic energy & $160 \mathrm{MeV}$ \\
Extraction kinetic energy & $2 \mathrm{GeV}$ \\
Circumference & $1 / 4 \mathrm{C}_{\mathrm{PS}} \approx 157.08 \mathrm{~m}$ \\
Repetition rate & $0.8 \mathrm{~Hz}$ \\
Cell type & Triplet \\
Number of cells & 16 \\
Number of inserted straight & 0 \\
$\quad$ sections & 16 \\
Symmetry & $Q_{x}=4.28, Q_{y}=4.55$ \\
Working point & 4.03 \\
Gamma transition & $H^{-}$charge exchange \\
Injection & injection \\
& $V_{\mathrm{rf}}(h=1)=8 \mathrm{kV}$, \\
rf system & $V_{\mathrm{rf}}(h=2)=6 \mathrm{kV}$ \\
& \\
\hline \hline
\end{tabular}

\section{B. RCS optics}

The first lattice and optics for a very compact RCS with only $1 / 7$ th of the PS circumference was proposed at the Chamonix workshop 2011 [11]. As suspected already at this stage of the study, the space available for correction magnets, instrumentation and other equipment proved to be insufficient and the circumference was then increased to 4/21th of the PS circumference, implying an operation of the RCS with harmonic number $h=4$ or $h=1$. The following feasibility study was performed for $h=1$ keeping $h=4$ as a possible option [12]. The main parameters used for the feasibility study are listed in Table II.

Due to the still small circumference, a high dipole filling factor was required suggesting a FODO structure and a threefold symmetry with three arcs and three straight sections for injection, extraction and rf. The number of cells was chosen as a compromise between a high enough gamma transition $\left(\gamma_{\mathrm{T}}>\gamma_{\text {extraction }}=3.13\right)$, and a still acceptable phase advance per cell. As the preferred lattice, a 21-cell FODO lattice with three straight sections and a dispersion suppression over a phase advance of $2 \pi$ had

TABLE II. RCS parameters as proposed in the feasibility study [12].

\begin{tabular}{lc}
\hline Injection kinetic energy & $160 \mathrm{MeV}$ \\
Extraction kinetic energy & $2 \mathrm{GeV}$ \\
Circumference & $4 / 21 \mathrm{C}_{\mathrm{PS}} \approx 119.68 \mathrm{~m}$ \\
Repetition rate & $10 \mathrm{~Hz}$ \\
Cell type & FODO \\
Number of cells & 21 \\
Number of inserted & 3 \\
$\quad$ straight sections & 3 \\
Symmetry & $4<Q_{x}, Q_{y}<5$ \\
Working point & $>3.6$ \\
Gamma transition & \\
Injection & $H^{-}$charge exchange injection \\
rf system & $V_{\mathrm{rf}}(h=1$ to 4$)=60 \mathrm{kV}$ \\
\hline \hline
\end{tabular}




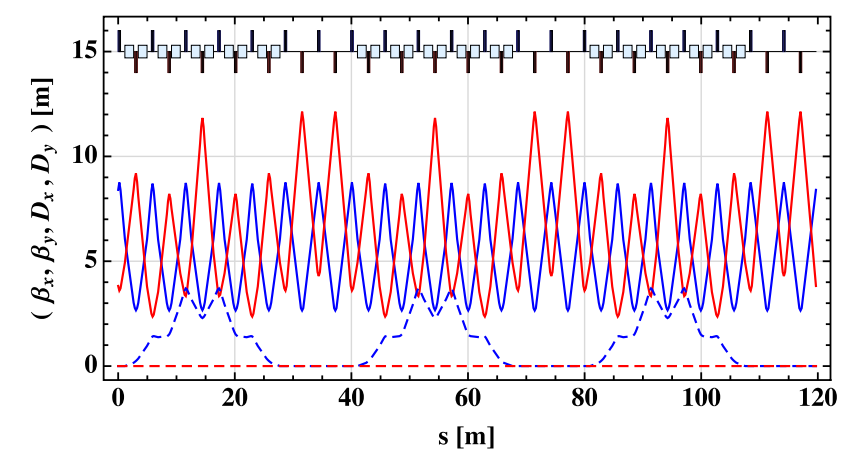

FIG. 2. RCS lattice and optics as proposed in the feasibility study [12].

been chosen, of which the optics are shown in Fig. 2. This choice had been based on civil engineering considerations and space requirements, but not on an optimization of the lattice and optics. A detailed description of all proposed lattices can be found in [13].

\section{Comparison of PS Booster and RCS optics}

Comparing the PS Booster and RCS lattice, the PS Booster lattice and optics in general appears to be very regular and smooth. The main reason is that, in the case of the RCS lattice, the symmetry is broken by the inserted straight sections contrary to the PS Booster lattice.

Coming back to the lattice and optics characteristics described in Sec. I, the PS Booster features a small variation of the beam size and a high symmetry of the lattice and optics, whereas the proposed RCS optics exhibits a smaller phase advance per cell. Furthermore, the RCS requires a dispersion suppression scheme in order to ensure zero or small dispersion in the straight sections.

\section{PARAMETERS, SIMULATION METHOD AND SETTINGS}

In this section the beam and lattice parameters used for the simulations (Sec. III A) and the simulation method and settings itself (Sec. III B) are summarized.

\section{A. General beam and lattice parameters}

Due to their small emittance, the ultimate LHC $25 \mathrm{~ns}$ beams [14] required for the HL-LHC upgrade [5] are most challenging with respect to the space-charge limit. These beam parameters are therefore used as baseline parameters for the simulations presented in this paper (Table III).

The standard assumption for the ultimate LHC $25 \mathrm{~ns}$ beam is a parabolic bunch shape with a bunch length of $180 \mathrm{~ns}$ at $2 \mathrm{GeV}$ and a longitudinal emittance of $\epsilon_{\text {long }}=5 \pi \sigma_{E} \sigma_{t}=2 \mathrm{eVs}$. However, for a PS Boosterlike machine a parabolic squared distribution in the longitudinal — and also transverse plane - is more realistic. To obtain the corresponding parameters leading to the same bunch length of $180 \mathrm{~ns}$, but under the assumption
TABLE III. Ultimate LHC $25 \mathrm{~ns}$ beam parameters used for simulations. The longitudinal emittance is rescaled as a parabolic squared distribution is assumed instead of a parabolic distribution.
Number of particles per bunch

Horizontal/vertical normalized emittance

Longitudinal emittance
$2.4 \times 10^{12}$

$2.5 / 2.5 \mathrm{~mm} \mathrm{mrad}$ $2.8 \mathrm{eVs}$ of a parabolic squared distribution with $\epsilon_{\text {long }}=7 \pi \sigma_{E} \sigma_{t}$, the emittance can simply be rescaled, leading to $\epsilon_{\text {long }}=\frac{7}{5} \times 2 \mathrm{eVs}=2.8 \mathrm{eVs}$.

The rf parameters are chosen in order to fit the RCS cycle of $10 \mathrm{~Hz}$ and the desired longitudinal emittance of $2.8 \mathrm{eVs}$ leading to $V_{\mathrm{rf}}=50 \mathrm{kV}$. To have as simple a system as possible, the double harmonic rf system foreseen for the RCS, and also present in the existing PS Booster, is replaced by a single harmonic system with $h=1$.

Furthermore, the proposed RCS lattices all had a circumference of 4/21th of the PS circumference. For the following more general studies, the circumference is increased to the PS Booster circumference of 1/4th of the PS circumference in order to make a direct comparison with the PS Booster lattice possible, and also to regain more flexibility in the lattice and optics. In addition, the magnet length of all lattices is adjusted, so that all lattices have approximately the same dipole strength and quadrupole gradient. The resulting machine parameters used for simulations are summarized in Table IV.

In general, it should be kept in mind that for the general studies presented in this paper, which have the aim of studying the influence of the space-charge effect, the above simplifications can and should be made, but that these lattices do not represent realistic machines.

\section{B. Simulation method and settings}

For the comparison of the different lattices, the parallel particle in cell (PIC) routines of the code PTC-ORBIT were used [15]. PTC-ORBIT combines the fully symplectic polymorphic tracking code PTC [16] with the well tested space-charge calculation routines of ORBIT [17]. The general simulation method is to define regularly spaced nodes along the lattice, at which the space-charge field of the beam is calculated and the resulting kick then applied to

TABLE IV. Lattice and optics parameters used for simulations.

\begin{tabular}{lc}
\hline \hline Injection kinetic energy & $160 \mathrm{MeV}$ \\
Extraction kinetic energy & $2 \mathrm{GeV}$ \\
Circumference & $1 / 4 \mathrm{C}_{\mathrm{PS}} \approx 157.08 \mathrm{~m}$ \\
Repetition rate & $10 \mathrm{~Hz}$ \\
Injection & $H^{-}$charge exchange injection \\
rf system & $V_{\mathrm{rf}}(h=1)=50 \mathrm{kV}$ \\
\hline \hline
\end{tabular}


TABLE V. Simulation parameters.

Number of macroparticles

Number of longitudinal bins

Number of bins for transverse mesh

Distance between space-charge nodes

the beam distribution. In between the space-charge nodes the beam is tracked with PTC.

In order to keep the simulation time reasonable, the socalled $2 \frac{1}{2} \mathrm{D}$ model has been used for the calculation of the space-charge field in the case of all simulations presented in this paper. With this method, all particles, independent of their longitudinal position, are projected into the transverse plane. The transverse space-charge field is then calculated for the projected distribution and afterwards scaled with the longitudinal distribution.

As is the case with PIC codes in general, the simulations become dependent on the number of macroparticles, the grid size and the number of space-charge kicks. A convergence study was performed [13] in order to find an adequate set of simulation parameters summarized in Table V.

All initial distributions have been matched in 6D to the Twiss parameters of the unperturbed optics and rf parameters of the lattice. The initial small oscillations visible in the time evolution of the emittances (e.g., Fig. 5) are due to the small mismatch between the Twiss parameters of the unperturbed optics and the real Twiss parameters including the space-charge effects.

The overall simulation time can be further reduced by optimizing the lattice description used for PTC. This optimization is as well described in detail in [13].

For all studies presented in this paper the initial beam distribution has been tracked for 10000 turns. This choice is based on the following considerations: Assuming a cycle of $10 \mathrm{~Hz}$ and a linear ramp-up for $60 \%$ of the cycle and rampdown for $40 \%$ of the cycle, the full ramp-up would take about 100000 turns. A simulation of the full ramp-up is therefore unrealistic due to excessive computing requirements. Taking into account that the space-charge force scales with $1 /\left(\beta \gamma^{2}\right)$, the space-charge effect is most relevant at low energy and it is sufficient to simulate only a smaller number of turns with realistic CPU time requirements. Therefore 10000 turns, corresponding to approximately 40 synchrotron periods, have been used.

To compare the different lattices more quantitatively, the relative emittance growth is compared, which we define in this paper by

$$
\frac{\Delta \epsilon_{z}}{\epsilon_{z, 0}}=\frac{\epsilon_{z}(n)-\epsilon_{z, 0}}{\epsilon_{z, 0}}, \quad z=x, y
$$

where $n$ is the number of turns tracked with $n=9700$ in this paper. 9700 turns have been chosen as not all simulations finished the 10000 turns due to issues with the simulation cluster.

Furthermore, for a better comparison with lattices with a threefold symmetry like the RCS lattice proposed in the feasibility study (Fig. 2), the working point has been decreased for most lattices by an integer with respect to the PS Booster working point, explicitly to $Q_{x} / Q_{y}=$ $4.28 / 3.55$. In the case of a threefold symmetry, the $2 Q_{y}=$ 9 resonance is systematic, and thus excited for a working point of $Q_{y}=4.55$, while for the regular 16-cell lattices with a 16-fold symmetry the resonance is nonsystematic and attenuated (see Sec. IV C for the influence of systematic and nonsystematic resonances).

\section{Estimate of emittance growth due to numerical noise}

The simulations presented in this paper have been conducted using a PIC code known to introduce numerical noise leading to artificial emittance growth. In order to get an estimate for the expected artificial emittance growth due to numerical noise and thereby an adequate set of simulation parameters, a convergence study has been conducted. In this study the dependence of the emittance evolution on the number of macroparticles, the number of longitudinal bins and the distance between space-charge nodes has been studied using the regular 16-cell triplet lattice (Fig. 4) as the reference lattice and for half the expected beam intensity, explicitly $1.2 \times 10^{12}$ particles per bunch. Similar results are expected for the other lattices presented in this paper. The beam has been tracked over 2000 turns, corresponding to approximately eight synchrotron periods, which is considered to be sufficient time to test the stability of the beam. As the artificial emittance growth due to numerical noise usually depends linearly on the number of turns [13], the emittance evolution can be scaled linearly to 10000 turns and an estimate for the emittance growth obtained.

For the simulation parameters used for the studies presented in this paper (Table V) but half the intensity$1.2 \times 10^{12}$ per bunch-the convergence study [13] yields a relative artificial emittance growth of

$$
\begin{aligned}
& \frac{\Delta \epsilon_{x}}{\epsilon_{x, 0}}=\frac{\epsilon_{x}(n=9700)-\epsilon_{x, 0}}{\epsilon_{x, 0}}=2.45 \times 10^{-3}, \\
& \frac{\Delta \epsilon_{y}}{\epsilon_{y, 0}}=\frac{\epsilon_{y}(n=9700)-\epsilon_{y, 0}}{\epsilon_{y, 0}}=5.21 \times 10^{-3},
\end{aligned}
$$

where the linear extrapolation to 9700 turns has been used for the calculation of $\Delta \epsilon_{x}$ :

$$
\begin{aligned}
& \epsilon_{x}(n)=4.11175 \mu \mathrm{m}+0.78 \times 10^{-6} \times n \frac{\mu \mathrm{m}}{\text { turn }}, \\
& \epsilon_{y}(n)=4.11270 \mu \mathrm{m}+1.85 \times 10^{-6} \times n \frac{\mu \mathrm{m}}{\text { turn }},
\end{aligned}
$$


where $n$ is the turn number and $\epsilon_{x / y, 0}=4.10924 \mu \mathrm{m}$ corresponds to $2.5 \mu \mathrm{m}$ normalized emittance.

In order to get an estimate for the beam intensity used in the simulations, the estimates for $1.2 \times 10^{12}$ per bunch have to be scaled to double the intensity of $2.4 \times 10^{12}$. It has been shown that the relative artificial emittance growth due to numerical noise increases proportional to $N_{b}^{2}$, where $N_{b}$ is the number of particles per bunch [18]. The relative emittance growth for $2.4 \times 10^{12}$ is thus just 4 times the relative emittance growth for $1.2 \times 10^{12}$ per bunch:

$$
\begin{aligned}
& \frac{\Delta \epsilon_{x}}{\epsilon_{x, 0}}\left(N_{b}=2.4 \times 10^{12}\right)=0.98 \times 10^{-2}, \\
& \frac{\Delta \epsilon_{y}}{\epsilon_{y, 0}}\left(N_{b}=2.4 \times 10^{12}\right)=2.08 \times 10^{-2} .
\end{aligned}
$$

\section{LATTICE STUDIES}

Before starting the discussion of the simulation results, we would like to emphasize that this paper aims at finding lattice and optics characteristics advantageous in the case of strong space-charge effects. We explicitly do not investigate different mechanisms responsible for emittance growth or the effect of the injection process. In most cases the observed emittance growth could be interpreted by the excitation of coherent modes as described in [19], but another explanation might as well be found and equally valid.

Furthermore, the lattices studied in this paper consist of only dipole and quadrupole magnets, and the optics are thus purely linear. The lattices do thus not contain any sextupoles for chromatic corrections. As in the case of the lattices and optics presented in this paper, the detuning with momentum is small compared to the tune spread due to space-charge, any effect due to the uncorrected chromaticity is therefore likewise expected to be small.

\section{A. Influence of fringe fields}

For realistic scenarios, and in particular for compact machines with short magnets, it is important to take the effect of fringe fields into account. In general, and as implemented in PTC-ORBIT, fringe fields do not perturb the linear optics, but introduce nonlinear, e.g., sextupolar and octupolar-like, perturbations.

As mentioned earlier, all lattices used for the presented studies only include dipoles and quadrupoles. The effect of the dipole fringe field is usually small and can be neglected, while the effect of the quadrupole fringe field can be considerable in some cases. To give an example of the influence of the quadrupole fringe fields, the time evolution of the emittances for two 16-cell lattices is shown in Fig. 3, where the first lattice consists of triplet and the second of "collapsed" triplet cells (see Fig. 6 for the optics). The two lattices are almost equivalent and also lead to almost equivalent linear optics. Without quadrupole fringe fields,
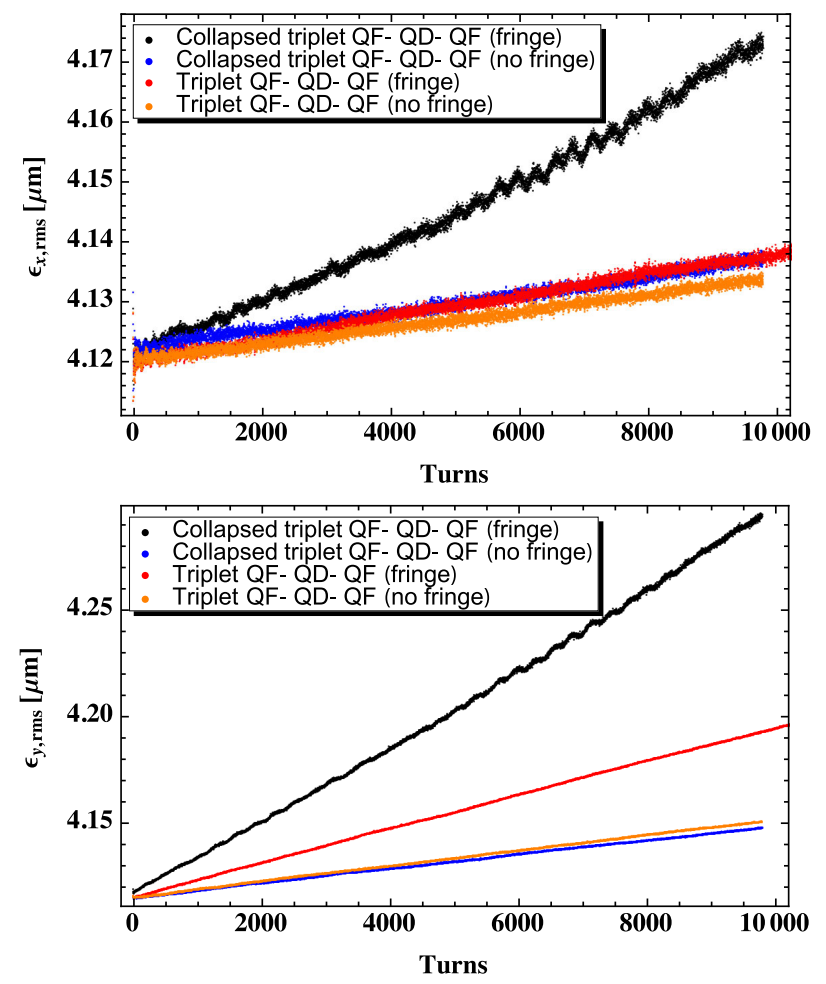

FIG. 3. Time evolution of horizontal (top) and vertical (bottom) rms emittance for the triplet and collapsed triplet cell lattice including fringe fields (fringe) and for the triplet cell lattice without fringe fields.

the difference in the time evolution of the emittances is small, while in the presence of quadrupole fringe fields, clearly a larger emittance growth is seen for the lattice with the stronger quadrupole fringe fields, here in the case of the collapsed triplet cells (Fig. 3).

The example shows that the effect of the quadrupole fringe fields can be dominant over the original effect of interest. Therefore, in order to further isolate the influence of the space-charge effect on the linear optics, no fringe fields except the very weak dipole fringe fields have been included. In PTC the dipole fringe field cannot be disabled, but its effect is expected to be negligible.

\section{B. Dependence on the lattice cell and variation of the beam size}

Many modern lattices are based on a FODO cell structure, which exhibits a relatively large variation of the beam size compared to a doublet or triplet structure. Roughly speaking, a very smooth variation of the beam size, and in particular small variations of the ratio between the two transverse planes, results in an almost uniformly distributed space-charge field. A large variation of the beam size on the other hand entails a varying strength of the nonlinear space-charge kicks, and the latter can therefore lead to excitation of resonances and stronger emittance growth. 

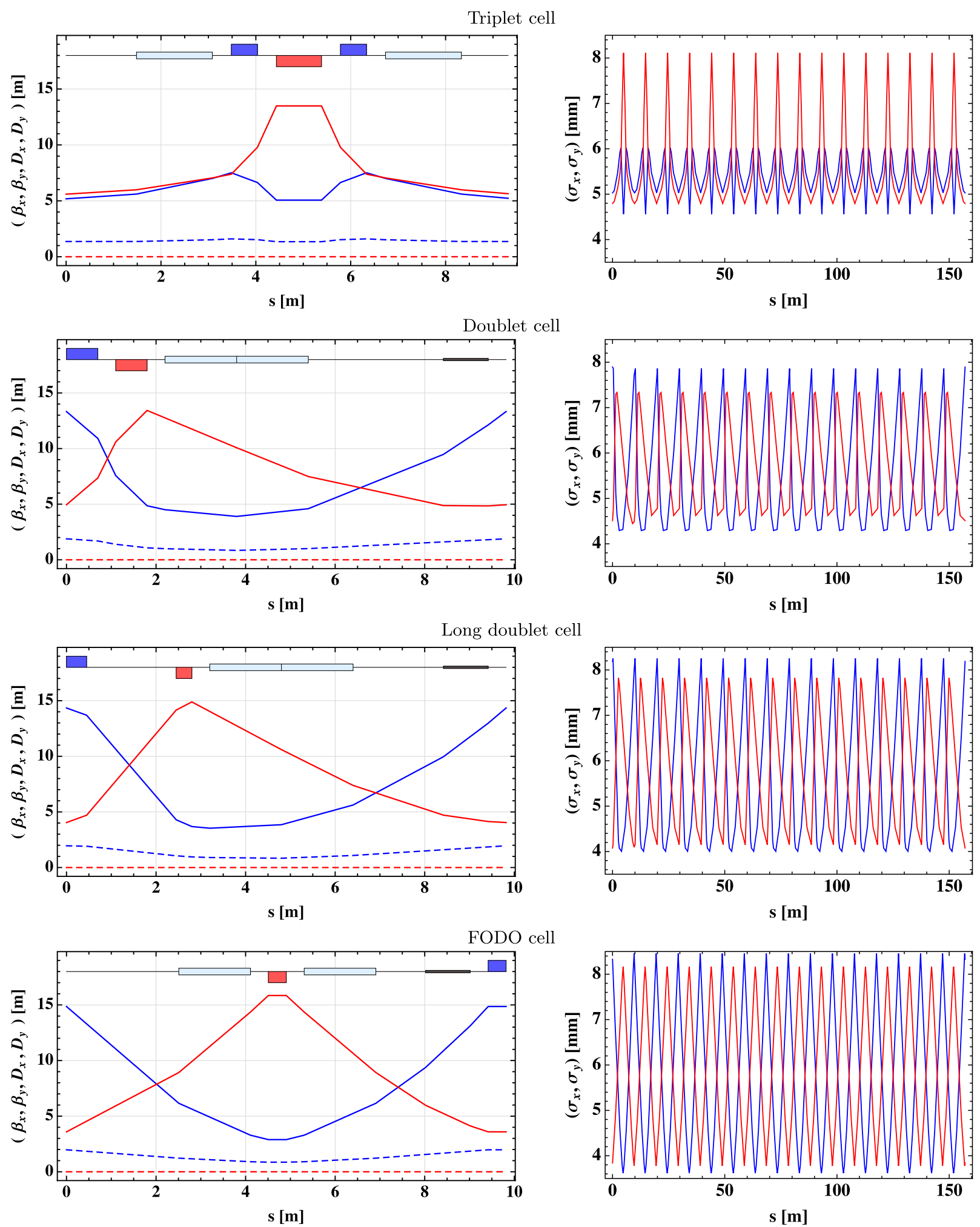

FIG. 4. Optics (left) of the different lattice cells and one rms beam envelope of the complete lattice (right). From top to bottom: triplet, doublet, long doublet and FODO. The beam envelope has been calculated using the design transverse normalized rms emittance of $2.5 \mu \mathrm{m}$ and the rms momentum spread corresponding to the design longitudinal emittance of $2.8 \mathrm{eVs}$. 
To investigate this, different 16 equal cell lattices with a working point of $Q_{x} / Q_{y}=4.28 / 3.55$ have been compared.

\section{Comparison of triplet, doublet, long doublet and FODO cell lattices}

Regular triplet cell lattices as used for the PS Booster feature the least variation of the beam size in comparison with the doublet, the long doublet (doublet with a larger distance between the quadrupoles), and finally the FODO cell lattices with the largest variation (Fig. 4 and Table VI).

The simulation results show (Fig. 5 and Table VI) that indeed the least rms emittance growth in the vertical plane is observed for the triplet lattice, followed by the doublet, while the long-doublet and FODO cell are comparable. This order is strictly speaking not valid for the triplet lattice in the vertical plane, in which case the doublet lattice features the smallest $\Delta \sigma_{y}$. However, and as will be shown in Sec. IV B 2, this larger variation only occurs over a small length and for this reason does have a comparably smaller effect.

\section{Comparison of triplet and collapsed triplet cell lattices}

Comparing the difference between the maximum and minimum beam size of the triplet, doublet, long doublet and FODO cell lattices of Sec. IV B 1, the triplet cell shows the smallest difference in the horizontal plane, but a large difference in the vertical plane. This large difference occurs only over a small length and seems to be of little importance, while for the doublet, long doublet and FODO lattice the large variation is not limited to a small section and leads to a stronger emittance growth. This observation indicates that not only the maximum variation of the beam size plays a role, but also the length of the section with the large variation.

To study this observation, a regular 16-cell lattice consisting of triplet cells collapsed to almost zero length has been compared to a regular 16-cell triplet lattice (the simplified PS Booster lattice). Both optics are shown in Fig. 6. In particular, the section with a high vertical beta function has been shortened in the case of the collapsed

TABLE VI. Maximum and minimum rms beam size and relative emittance growth in $\mathrm{mm}$ for triplet, doublet, long doublet and FODO lattice, where the notation $\Delta \sigma_{x / y}=\sigma_{x / y \text {,max }}-\sigma_{x / y \text {,min }}$ was used. The beam size has been calculated using the design transverse normalized rms emittance of $2.5 \mu \mathrm{m}$ and the rms momentum spread corresponding to the design longitudinal emittance of $2.8 \mathrm{eVs}$.

\begin{tabular}{lcccc}
\hline \hline Cell type & $\Delta \sigma_{x}$ & $\Delta \sigma_{y}$ & $\frac{\Delta \epsilon_{x}}{\epsilon_{x, 0}}\left[10^{-2}\right]$ & $\frac{\Delta \epsilon_{y}}{\epsilon_{y, 0}}\left[10^{-2}\right]$ \\
\hline Triplet & 1.46 & 3.32 & 0.58 & 1.00 \\
Doublet & 3.60 & 2.89 & 0.78 & 1.31 \\
Long doublet & 4.26 & 3.74 & 0.65 & 1.60 \\
FODO & 4.84 & 4.39 & 0.66 & 1.61 \\
\hline \hline
\end{tabular}
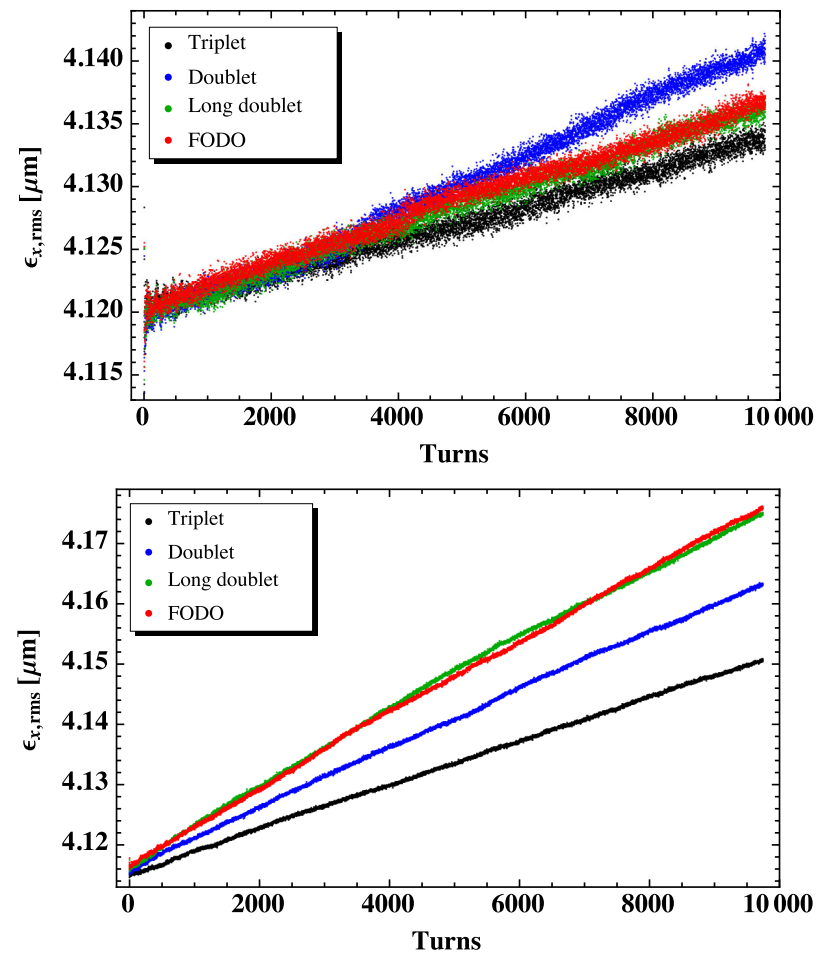

FIG. 5. Time evolution of horizontal (top) and vertical (bottom) rms emittance for different lattice cells.

triplet cell and with it the length of the section with a large variation of the beam size.

The simulation results shown in Fig. 7 confirm the above hypothesis: the emittance growth in the vertical plane for
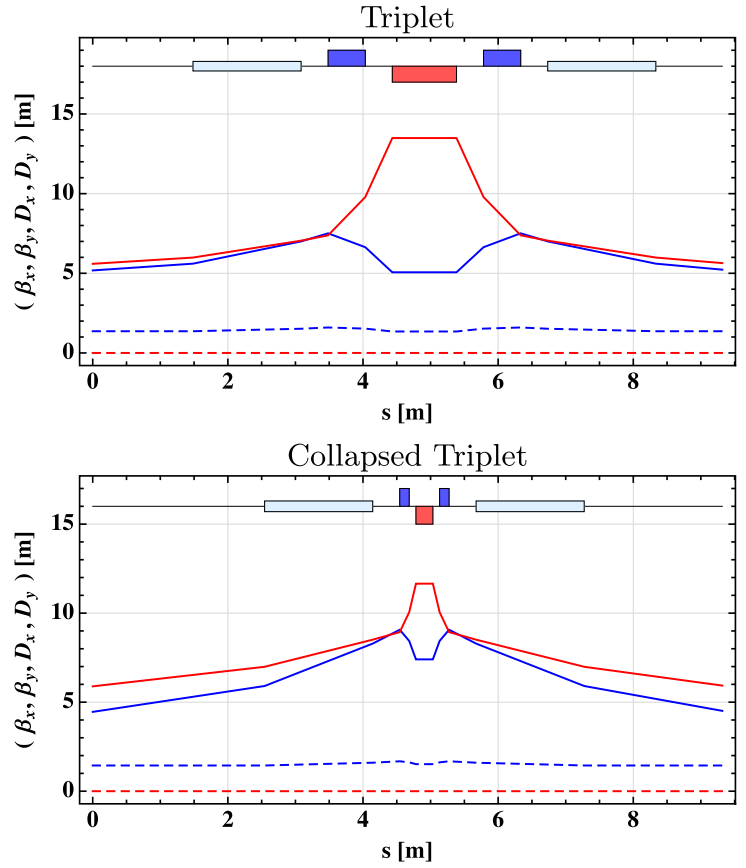

FIG. 6. Optics of the triplet (top) and collapsed triplet (bottom) cell lattice. 

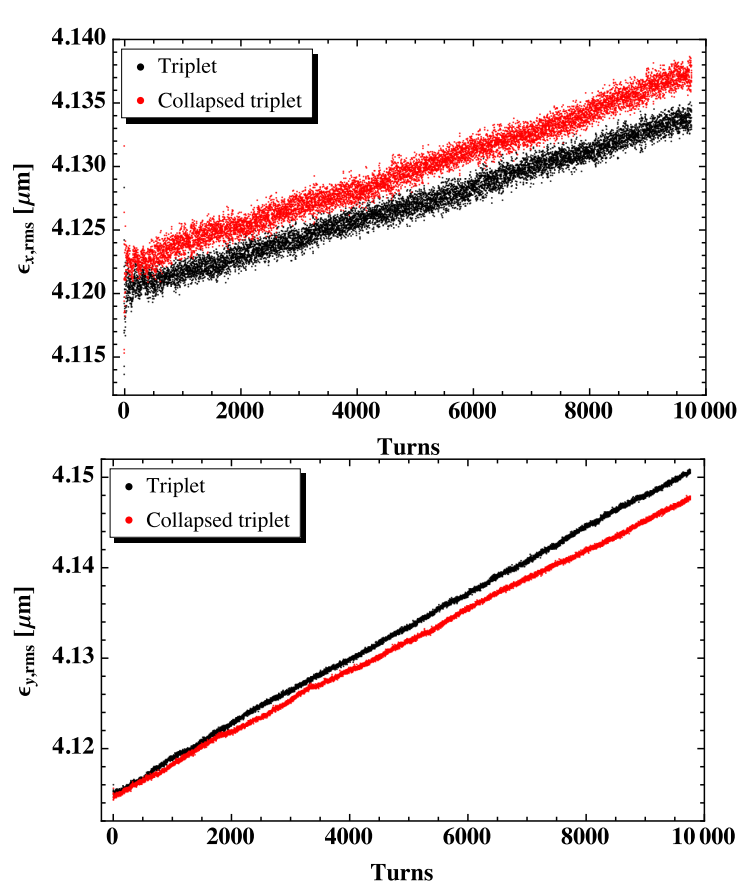

FIG. 7. Time evolution of horizontal (top) and vertical (bottom) rms emittance for the triplet and collapsed triplet cell lattice.

the collapsed triplet is slightly less than for the triplet, while the horizontal plane stays unchanged.

\section{Conclusion}

Comparing lattices that only differ in their cell types, a smoother focusing and small variation of the beam size is advantageous. In addition, the length of the section with a large variation is of importance. If the large variation of the beam size is very localized, the emittance growth stays small. However, one has to keep in mind that this is in general achieved with a large number of strong focusing magnets. Consequently, the beneficial effect of the small variation of the beam size could then be already canceled by the effect of the fringe fields.

\section{Influence of systematic resonances}

Comparing the PS Booster lattice and the lattices proposed for the RCS and other high power hadron machines [8-10], the RCS lattices are characterized by a low twofold, threefold or fourfold symmetry in contrast to the 16-fold symmetry of the PS Booster lattice [13]. The lattice symmetry in general defines the systematic and nonsystematic resonances, where the systematic resonances for a lattice consisting of $N$ superperiods are given by

$$
m Q_{x}+n Q_{y}=p N, \quad m, n, p \in \mathbb{Z} .
$$

Based on this observation, we want to address the question of the relevance of the lattice symmetry defining the systematic resonances by studying two lattices: (i) a 16-cell triplet lattice, where the symmetry of the lattice is broken by shortening the bends in one, two or four cells (Sec. IV C 1) and (ii) a FODO lattice with different numbers of cells, explicitly 15, 16, 18, 21 and 24 cells (Sec. IV C 2).

Both cases are motivated by questions raised in the context of the PS Booster $2 \mathrm{GeV}$ energy upgrade and the RCS design study. An example for the first case is the proposal of shortening the bends in one lattice cell in the PS Booster in order to enlarge the section for the $\mathrm{H}^{-}$injection. The second case addresses the question of the best number of lattice cells raised at the very beginning of the design of the RCS. The standard arguments in this context are the compactness of the machine, favoring a small number of cells, and, by contrast, a low phase advance per cell, favoring a large number of cells. A small phase advance per cell is favored, as the variation of the beam size increases with increasing phase advance per cell. By lowering the tune, the cell length can certainly be shortened without increasing the phase advance per cell, but in case the machine has to be operated below gamma transition, as, e.g., in the case of the RCS, the lowest value of the tune is limited by the requirement of $\gamma_{\text {extraction }}<\gamma_{\mathrm{T}}$ and $\gamma_{T} \approx Q_{x}$. However, from the simulations presented in this section, it turns out that the phase advance per cell does not have a very large impact on the emittance growth in comparison to the influence of the lattice symmetry, which can in general influence the emittance growth and thus machine performance considerably.

\section{Comparison of 16-cell triplet lattices with broken symmetry}

Starting from the regular 16-cell triplet lattice (the simplified PS Booster lattice) with a working point of $Q_{x} / Q_{y}=4.28 / 3.55$, the symmetry of the lattice is broken by shortening the bends in one triplet cell leading to a lattice with a onefold symmetry. Due to the edge focusing of the bending magnets, the shorter bends introduce vertical beta-beating and a tune change. The beta-beating is corrected by matching the modified cell to the regular arc cells with the triplet next to the shortened bends. The tune is then adjusted by rematching the phase advance of the arc cells. Both adjustments are repeated until the target tune of $Q_{x} / Q_{y}=4.28 / 3.55$ is reached. The lattices with a twofold and fourfold symmetry are created in the same way, only that in this case the bends are shortened in two and four cells respectively. The optics of all four lattices are shown in Fig. 8.

The time evolution of the rms emittances of the four different lattices is plotted in Fig. 9 and compared in Table VII. As expected, the lattice with the lowest symmetry (onefold symmetry) shows the largest emittance growth. In the case of the lattice with a twofold symmetry the emittance growth is already considerably reduced and 

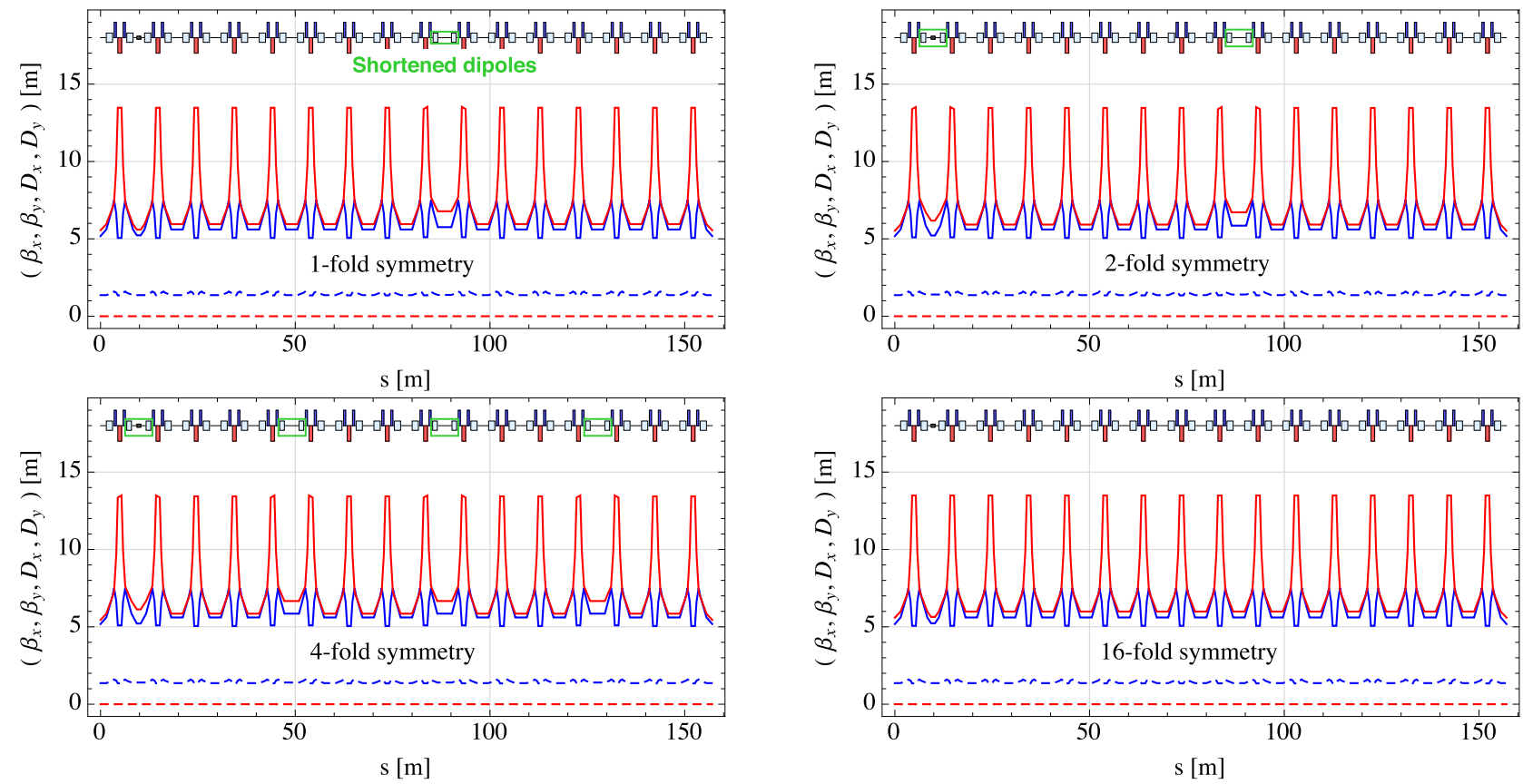

FIG. 8. Optics of the 16-cell triplet lattice with onefold, twofold, fourfold and complete 16-fold symmetry. The symmetry is broken by shortening the bends in the corresponding number of cells indicated in green.
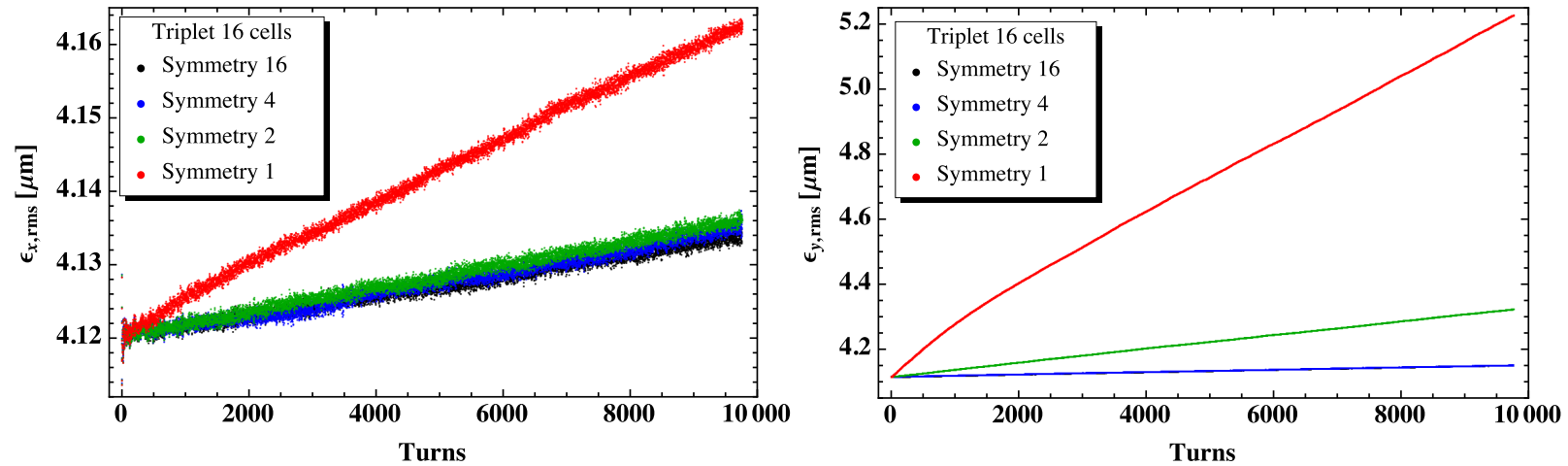

FIG. 9. Time evolution of horizontal (left) and vertical (right) rms emittance for the 16-cell triplet lattice with onefold, twofold, fourfold and 16-fold symmetry.

the lattices with fourfold and 16-fold symmetry exhibit the same time evolution of the emittances.

This behavior can be explained by an excitation of the $4 Q_{y}$ and $Q_{x}+4 Q_{y}$ resonance for the onefold and twofold but not the fourfold and 16-fold symmetry (Fig. 25). Both

TABLE VII. Relative emittance growth for the 16-cell triplet lattices with different symmetries.

\begin{tabular}{lcc}
\hline \hline Symmetry & $\frac{\Delta \epsilon_{x}}{\epsilon_{x, 0}}\left[10^{-2}\right]$ & $\frac{\Delta \epsilon_{y}}{\epsilon_{y, 0}}\left[10^{-2}\right]$ \\
\hline 16 & 0.58 & 1.00 \\
4 & 0.65 & 1.01 \\
2 & 0.66 & 5.16 \\
1 & 1.26 & 27.0 \\
\hline \hline
\end{tabular}

resonances are driven by space-charge as the nonlinearities due to the dipole fringe fields are small and no other nonlinearities, explicitly no errors, are included. In the case of the onefold and twofold symmetry both resonances are systematic, while for the fourfold and 16-fold symmetry both resonances are nonsystematic and thus the excitation suppressed.

\section{Comparison of regular FODO cell lattices with different number of cells}

In this section, regular FODO cell lattices consisting of different number of cells, explicitly 15, 16, 18, 21 and 24 are compared. The optics of all lattices have been matched to the same working point of $Q_{x} / Q_{y}=4.28 / 3.55$ as chosen also for the 16-cell triplet lattices of Sec. IV C 1. 

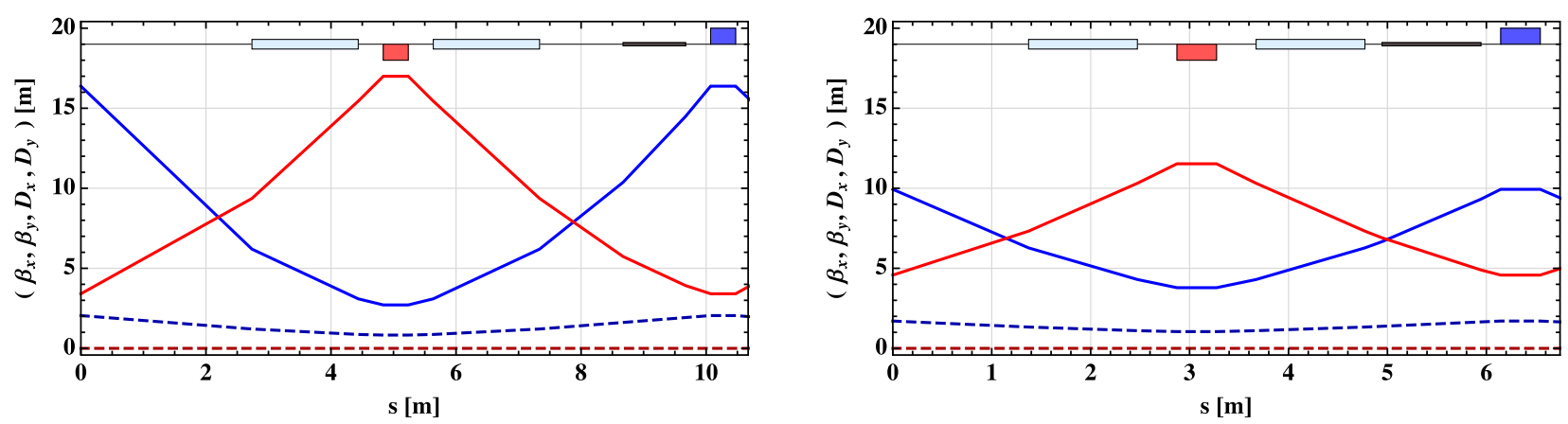

FIG. 10. Optics of one FODO cell for a regular FODO lattice consisting of 15 (left) and 24 (right) cells and a working point of $Q_{x} / Q_{y}=4.28 / 3.55$.
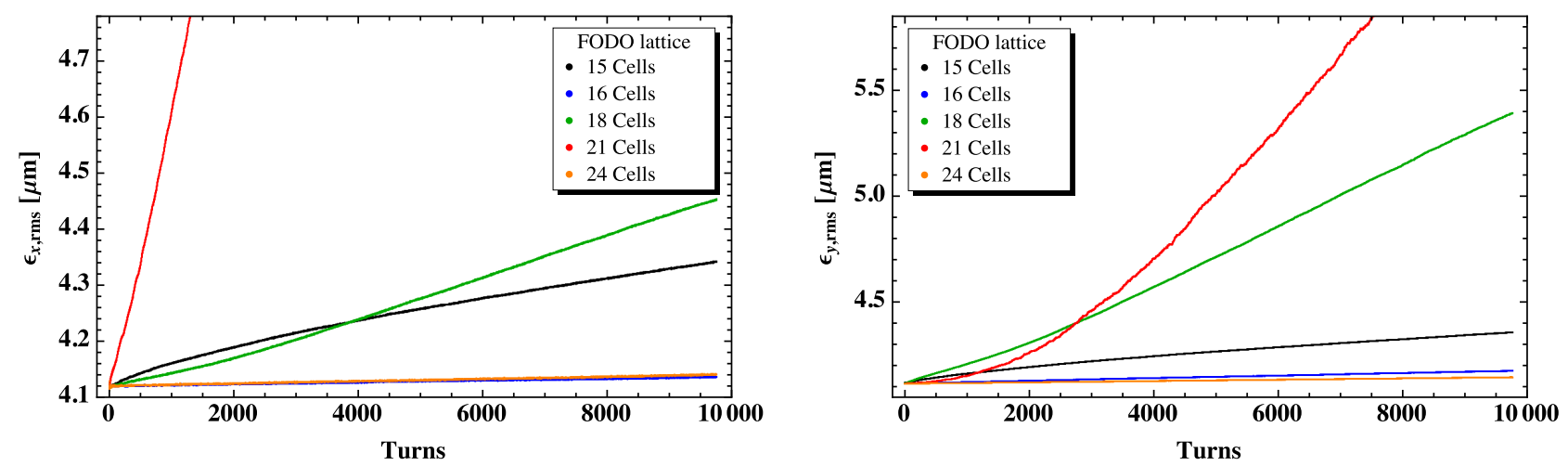

FIG. 11. Time evolution of horizontal (left) and vertical (right) rms emittance for the regular FODO cell lattices consisting of 15, 16, 18,21 and 24 cells.

Furthermore, the same dipole field has been assumed for all lattices and the dipole length adjusted accordingly. The length of the quadrupoles has been kept constant as the gradient does not change significantly for the different lattices. Representative for all regular FODO cell lattices, the optics of one lattice cell of the 15- and 24-cell FODO lattice is shown in Fig. 10.

As in the case of the regular triplet cell lattice with shortened bends (Sec. IV C 1), the time evolution of the emittances (Fig. 11) can be explained by the excitation of systematic resonances of 4th and 5th order driven by space-charge: For the FODO lattice with 15 cells the tune footprint crosses the $2 Q_{x}+2 Q_{y}$, for 18 cells the $Q_{x}+4 Q_{y}$

TABLE VIII. Relative emittance growth for regular FODO lattices with different number of cells. In this case the number of cells defines the lattice symmetry.

\begin{tabular}{lcc}
\hline \hline Number of cells & $\frac{\Delta \epsilon_{x}}{\epsilon_{x, 0}}\left[10^{-2}\right]$ & $\frac{\Delta \epsilon_{y}}{\epsilon_{y, 0}}\left[10^{-2}\right]$ \\
\hline 24 & 0.78 & 0.89 \\
16 & 0.66 & 1.61 \\
21 & 89.51 & 61.82 \\
18 & 8.30 & 31.05 \\
15 & 5.68 & 6.03 \\
\hline \hline
\end{tabular}

and for 21 cells the $5 Q_{x}$ resonance (Fig. 26). For 16 and 24 cells no systematic resonances are crossed and consistently the 16- and 24-cell lattice show a considerably smaller emittance growth than the lattices with 15, 18 and 21 cells (Table VIII).

\section{Conclusion}

Both examples - the triplet cell lattice where the symmetry is broken by introducing a variation of the beam size (Sec. IV C 1), and the regular FODO cell lattices with different number of cells, in which case the symmetry is defined by the number of lattice cells (Sec. IV C 2)-show that if the tune footprint crosses a systematic resonance an increased emittance growth is observed. Originally it was also suspected that the emittance growth would depend on the phase advance per cell, but in the case of the FODO cell lattice with different number of cells, the time evolution of the emittances is clearly dominated by the effect of the lattice symmetry.

\section{Weak and strong symmetry breaking}

In Sec. IV C 1 it has been shown that a breaking of the symmetry by introducing a variation of the beam size from the regular pattern at one or several locations, can lead to 
considerable emittance growth. In this section, we want to further investigate the influence of the variation of the beam size on the lattice symmetry, the excitation of resonances and ultimately the emittance growth by comparing different optics of the FODO cell lattices with a different number of lattice cells introduced in Sec. IV C 2.

At first, we want to study the case where only a small variation of the beam size is introduced for lattices with different symmetries (Sec. IV D 1), to which we will refer as "weak symmetry breaking," and investigate if the original lattice symmetry is of importance. As a second case, we will compare lattices with a large variation of the beam size- "strong symmetry breaking"-with the ones with only a small variation of the beam size-"weak symmetry breaking"-(Sec. IV D 2).

In all examples, the symmetry is broken by shortening the bends in one or several lattice cells. The introduced beta-beating is corrected by matching the Twiss functions of the cell with shorter bends to the arc cell using the individual quadrupoles adjacent to the shorter bends and afterwards adjusting the phase advance.

\section{Comparison of FODO cell lattices with weak symmetry breaking}

To study the effect of only a small deviation of the beam size from the regular pattern on the lattice symmetry, the FODO cell lattices with different number of cells of Sec. IV C 2 are compared, but in addition with shortened bends in one lattice cell leading to a onefold lattice symmetry for all lattices. Furthermore, the variation of the beam size introduced by the beta-beating is kept as small as possible and the optics are all matched to the same working point of $Q_{x} / Q_{y}=4.28 / 3.55$. Representatively for the beam envelope of the FODO cell lattices with a

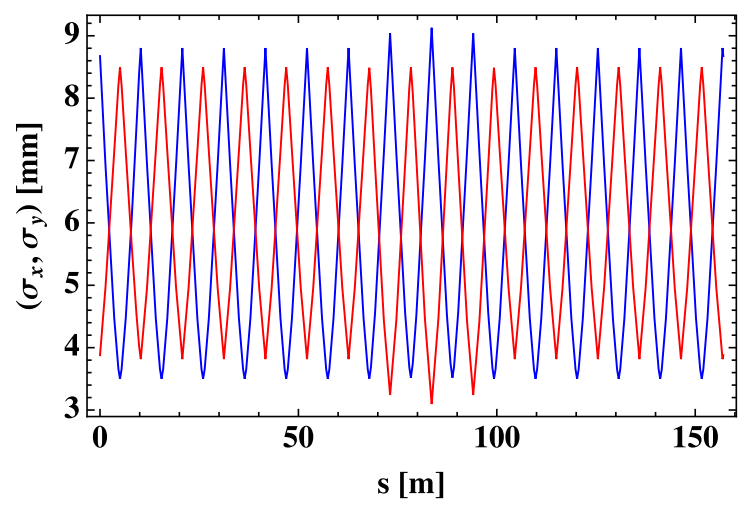

FIG. 12. One rms beam envelope for the 15-cell FODO lattice with a working point of $Q_{x} / Q_{y}=4.28 / 3.55$ and a onefold symmetry. The optics are matched keeping the variation of the beam size as small as possible. The beam envelope has been calculated using the design transverse normalized emittance of $2.5 \mu \mathrm{m}$ and the rms momentum spread corresponding to the design longitudinal emittance of $2.8 \mathrm{eVs}$.
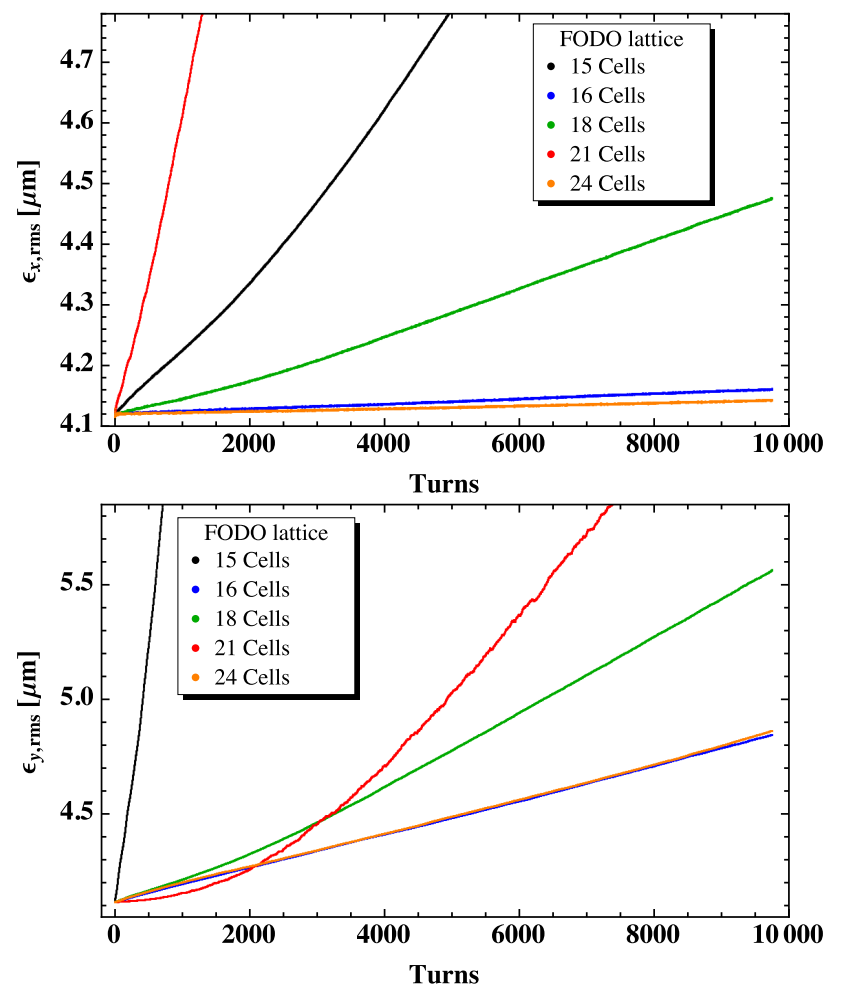

FIG. 13. Time evolution of horizontal (up) and vertical (bottom) rms emittance for a regular FODO lattice consisting of 15, $16,18,21$ and 24 cells and onefold symmetry.

different number of cells but one-fold symmetry, the beam envelope of the 15-cell FODO lattice is shown in Fig. 12.

As the bends are only shortened in one lattice cell, the lattice symmetry is reduced to a onefold symmetry. In case the symmetry would be "completely broken," the differences in the emittance growth due to the original lattice symmetry should disappear. However, comparing the time evolution of the emittances of the FODO lattices with weakly broken symmetry with the FODO lattices with unbroken symmetry (Figs. 13, Fig. 11, Table VIII, Table IX), the relative time evolution of the emittances of

TABLE IX. Relative emittance growth for regular FODO lattices with different number of cells (unbroken) and FODO lattices with different number of cells, but shortened bends in one lattice cell (broken). Here broken and unbroken refers to the breaking of the lattice symmetry by shortening the bends in one cell.

\begin{tabular}{lccccc}
\hline \hline & \multicolumn{2}{c}{ Unbroken } & & \multicolumn{2}{c}{ Broken } \\
\cline { 2 - 3 } \cline { 5 - 6 } Number of cells & $\frac{\Delta \epsilon_{x}}{\epsilon_{x, 0}}\left[10^{-2}\right]$ & $\frac{\Delta \epsilon_{y}}{\epsilon_{y, 0}}\left[10^{-2}\right]$ & & $\frac{\Delta \epsilon_{x}}{\epsilon_{x, 0}}\left[10^{-2}\right]$ & $\frac{\Delta \epsilon_{y}}{\epsilon_{y, 0}}\left[10^{-2}\right]$ \\
\hline 24 & 0.78 & 0.89 & & 0.81 & 18.19 \\
16 & 0.66 & 1.61 & & 1.25 & 17.80 \\
21 & 89.51 & 61.82 & & 90.78 & 64.11 \\
18 & 8.30 & 31.05 & & 8.85 & 35.07 \\
15 & 5.68 & 6.03 & & 35.02 & 1120.41 \\
\hline \hline
\end{tabular}


the different lattices with respect to each other is similar. This behavior suggests a remaining influence of the systematic resonances defined by the original lattice symmetry. Aside from the similar relative time evolution of the emittances, the emittance growth for the lattices with weakly broken symmetry is understandably visibly increased (Table IX).

\section{Comparison of FODO lattices with weak and strong symmetry breaking}

The example of the regular FODO lattice with a different number of cells and onefold symmetry showed that, if only a small perturbation of the regular pattern of the beam size is introduced by the shortened bends, the symmetry of the lattice is mostly preserved (Sec. IV D 1).

As a next step, we now want to test the influence of the perturbation on the breaking of the symmetry by comparing two different corrections of the beta-beating of the same lattice: one creating a small (Optics 1) and one a large (Optics 2) disturbance of the regularity of the beam envelope. As base lattice the regular 16-cell FODO lattice with a onefold symmetry and different working points is used. For a more quantitative measure of the deviation of the beam size and strength of the symmetry breaking, we define the deviation of the beam size $\Delta \sigma_{z}$ as the maximum deviation of the minimum/maximum of the beam size from the one of the regular cell:

$$
\Delta \sigma_{z}=\max \left(\sigma_{z, \max }, \sigma_{z, \min }\right), \quad z=x, y,
$$

with

$$
\begin{aligned}
& \Delta \sigma_{z, \max }=\max \left(\left|\left(\sigma_{z, \max }-\sigma_{\max , \text { cell }}\right)\right|\right), \\
& \Delta \sigma_{z, \min }=\max \left(\left|\left(\sigma_{z, \min }-\sigma_{\text {min,cell }}\right)\right|\right)
\end{aligned}
$$

where $\sigma_{z, \max }$ is the maximum of the beam size in one cell and $\sigma_{\max , \text { cell }}$ the maximum beam size in the unperturbed cell with the equivalent definition for the minimum.

Exemplary for all working points, the beam envelope of the two different optics for a working point of $Q_{x} / Q_{y}=$ 4.28/3.55 is shown in Fig. 14 and the time evolution of the

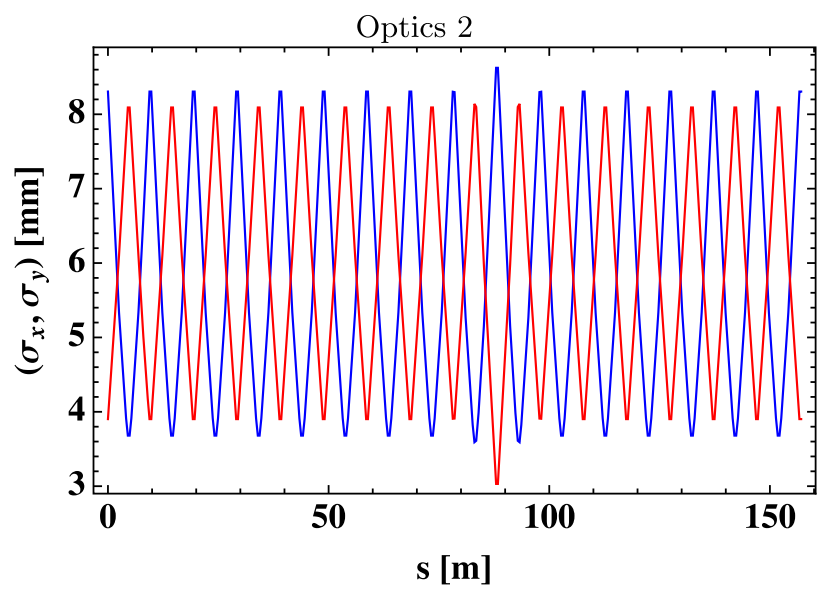

FIG. 14. One rms beam envelope of two different optics of the 16-cell FODO lattices with onefold symmetry and a working point of $Q_{x} / Q_{y}=4.28 / 3.55$ : Optics 1 (left) with a small variation of the regularity of the beam size and Optics 2 (right) with a large variation. The beam envelope has been calculated using the design transverse normalized rms emittance of $2.5 \mu \mathrm{m}$ and the rms momentum spread corresponding to the design longitudinal emittance of $2.8 \mathrm{eVs}$.
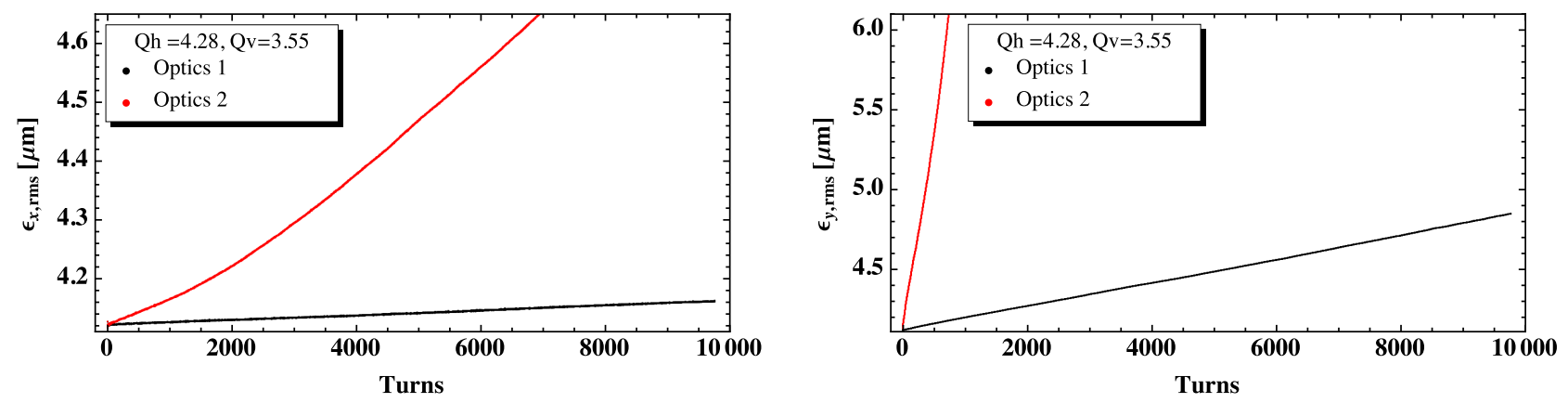

FIG. 15. Time evolution of horizontal (left) and vertical (right) rms emittance for Optics 1 and Optics 2 of the 16-cell FODO lattice with onefold symmetry and a working point of $Q_{x} / Q_{y}=4.28 / 3.55$. 
TABLE X. Deviation of the beam size $\Delta \sigma_{z}$ as defined in Eq. (9) and relative emittance growth for different matches (Optics 1, Optics 2 ) of the regular 16-cell FODO lattice with shortened bends in one lattice cell. If the emittance growth is marked with $\infty$, all particles were lost before reaching 10000 turns.

\begin{tabular}{|c|c|c|c|c|c|c|c|c|}
\hline \multirow[b]{2}{*}{$Q_{x / y}$} & \multicolumn{4}{|c|}{ Optics 1} & \multicolumn{4}{|c|}{ Optics 2} \\
\hline & $\Delta \sigma_{x}[\mathrm{~mm}]$ & $\Delta \sigma_{y}[\mathrm{~mm}]$ & $\frac{\Delta \epsilon_{x}}{\epsilon_{x, 0}}\left[10^{-2}\right]$ & $\frac{\Delta \epsilon_{y}}{\epsilon_{y, 0}}\left[10^{-2}\right]$ & $\Delta \sigma_{x}[\mathrm{~mm}]$ & $\Delta \sigma_{y}[\mathrm{~mm}]$ & $\frac{\Delta \epsilon_{x}}{\epsilon_{x, 0}}\left[10^{-2}\right]$ & $\frac{\Delta \epsilon_{y}}{\epsilon_{y, 0}}\left[10^{-2}\right]$ \\
\hline $2.28 / 1.55$ & 0.53 & 1.03 & 1.03 & 338.32 & 5.08 & 8.67 & 341.79 & 4343.72 \\
\hline $3.28 / 2.55$ & 0.11 & 0.32 & 7.60 & 18.82 & 1.75 & 3.04 & $\infty$ & $\infty$ \\
\hline $4.28 / 3.55$ & 0.07 & 0.10 & 1.25 & 17.80 & 0.32 & 0.87 & 19.61 & 1373.39 \\
\hline $5.28 / 4.55$ & 0.26 & 0.30 & 16.02 & 86.52 & 0.26 & 0.30 & 16.31 & 90.09 \\
\hline
\end{tabular}

emittances in Fig. 15. A comparison of the time evolution of the emittances of the two optics yields that already the slight difference in the matching results in a large increase of the emittance growth for Optics 2. This behavior is also observed for the other working points $Q_{x} / Q_{y}=2.28 / 1.55$ and $Q_{x} / Q_{y}=3.28 / 2.55$ (Table $\mathrm{X}$ ). As a last case, two different matches for the working point of $Q_{x} / Q_{y}=$ $5.28 / 4.55$ with a similar deviation of the beam size (both optics feature the same $\Delta \sigma_{z}$ ) are compared and yield a very similar emittance growth.

These four examples thus support further the hypothesis that the deviation of the beam size can be used as a measure for breaking of the lattice symmetry if different optics of one lattice and working point are compared.
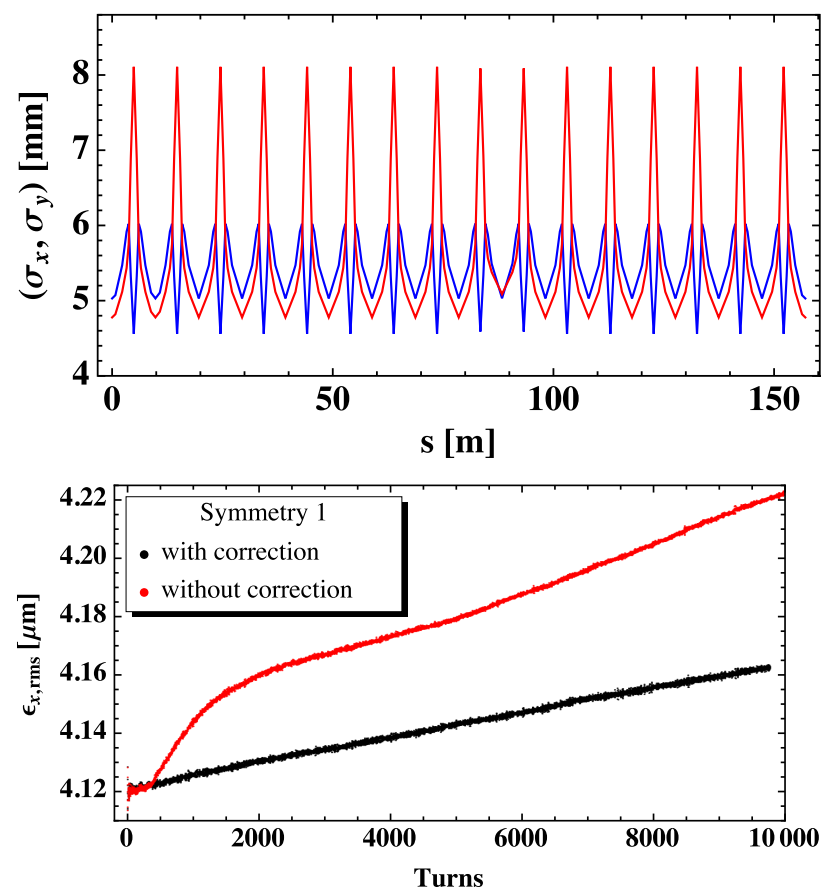

\section{Influence of beta-beating correction}

In all examples of symmetry breaking by shortening the dipoles in certain cells, the introduced beta-beating has been corrected by matching the modified cell to the normal arc cell with individual quadrupoles, and so minimizing the variation of the beam size and localizing the resulting irregular Twiss functions to the modified cell. The question addressed in this section is whether this correction is actually beneficial or not.

In the preceding sections it has been shown that the symmetry of the lattice, and also how the symmetry is broken, plays an important role. The examples presented in the following suggest that this also applies for the betabeating correction.
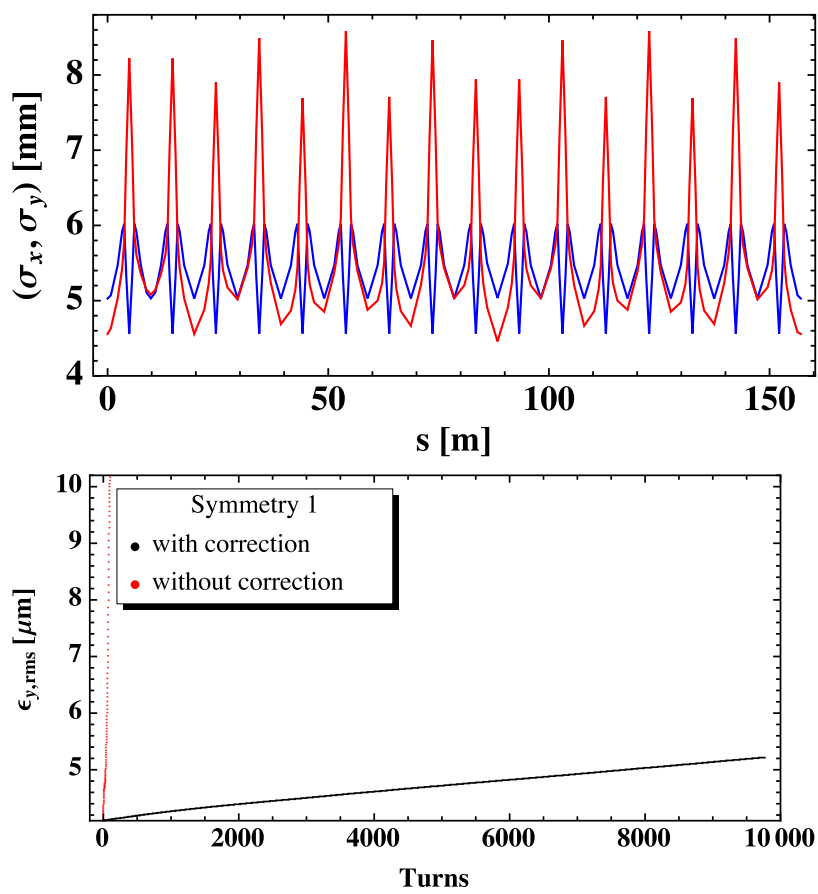

FIG. 16. One rms beam envelope (top) and time evolution of the emittance (bottom) of the regular 16-cell triplet lattice with onefold symmetry and a working point of $Q_{x} / Q_{y}=4.28 / 3.55$. The beam envelope has been calculated using the design transverse normalized rms emittance of $2.5 \mu \mathrm{m}$ and the rms momentum spread corresponding to the design longitudinal emittance of $2.8 \mathrm{eVs}$. The one rms beam envelope with beta-beating correction is shown on the upper left and without beta-beating correction on the upper right. The horizontal (left) and vertical (right) time evolution of the emittance for the lattice with and without beta-beating correction is shown on the bottom. 

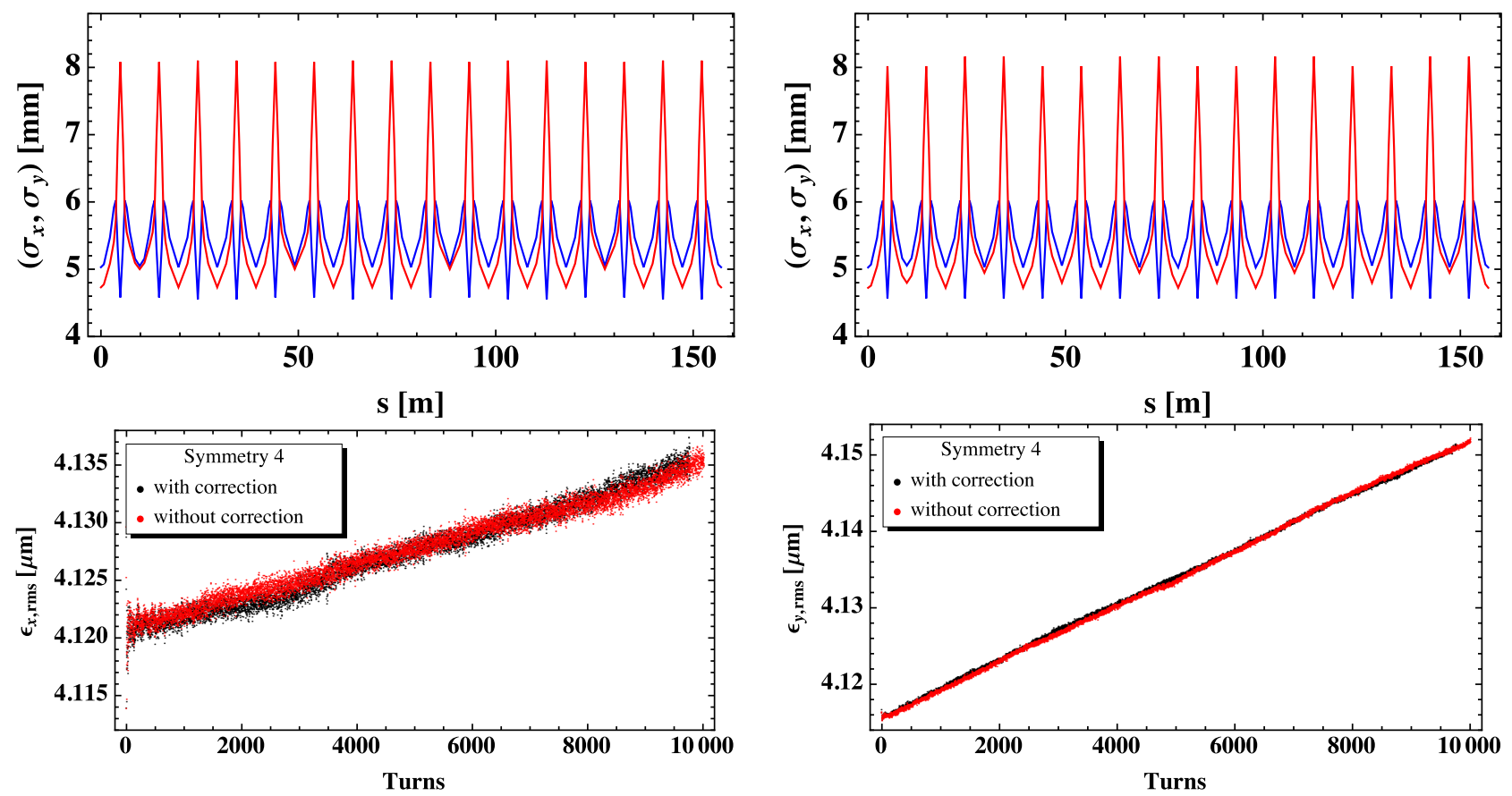

FIG. 17. One rms beam envelope (top) and time evolution of the emittance (bottom) of the regular 16-cell triplet lattice with fourfold symmetry and a working point of $Q_{x} / Q_{y}=4.28 / 3.55$. The beam envelope has been calculated using the design transverse normalized rms emittance of $2.5 \mu \mathrm{m}$ and the rms momentum spread corresponding to the design longitudinal emittance of $2.8 \mathrm{eVs}$. The one rms beam envelope with beta-beating correction is shown on the upper left and without beta-beating correction on the upper right. The horizontal (left) and vertical (right) time evolution of the emittance for the lattice with and without beta-beating correction is shown on the bottom.

As a first example, we compare the regular 16-cell triplet lattices with onefold symmetry and with and without betabeating correction (Fig. 16). In the case of the optics with beta-beating correction, the symmetry is only weakly broken. This weak breaking of the symmetry, in the case of the optics with beta-beating correction, then turns into a strong breaking of the symmetry, in the case without betabeating correction, resulting in turn in a larger emittance growth.

For comparison and as a second example, the betabeating correction in the case of the regular 16-cell triplet lattice with fourfold symmetry does not change the emittance growth. The reason is that for a fourfold symmetry no systematic resonances lie in the region of the tune footprint (see Fig. 25 for the resonance diagram). The introduced beta-beating preserves the fourfold symmetry of the lattice and thus both lattices feature the same time evolution of the emittance (Fig. 17). In addition, the introduced beta-beating is rather small and with it also the variation of the beam size.

\section{Conclusion}

The examples presented in Secs. IV D 1 and IV D 2 for weak and strong symmetry breaking and the example optics with and without beta-beating correction (Sec. IV D 3) suggest that in case of weak symmetry breaking, meaning that the disturbance of the regular pattern of the beam size is relatively small, the original symmetry of the lattice is only partly preserved resulting in a stronger emittance growth for lattices for which the tune footprint crosses a systematic resonance. In general, the stronger the disturbance of the regular pattern of the beam size, the stronger the symmetry is broken and the larger the emittance growth.

\section{E. Dependence on the phase advance per cell}

The initial motivation behind the example of a regular FODO lattice with different number of cells (Sec. IV C 2) and also the regular FODO lattice with a onefold symmetry and different working points (Sec. IV D 2) was not to study the effect of the lattice symmetry but to investigate the dependence of the emittance growth on the phase advance per cell. The expectation was that the emittance growth would increase with the phase advance per cell, but the examples showed that the effect of systematic resonances is dominating.

Nevertheless, in the case of the 16- and 24-cell regular FODO lattice (Sec. IV C 2) no systematic resonances are crossed and the two lattices can therefore be used as an example for the dependence on the phase advance per cell. The time evolution of the emittances for these two lattices is shown in Fig. 18, and indeed a smaller emittance growth is 

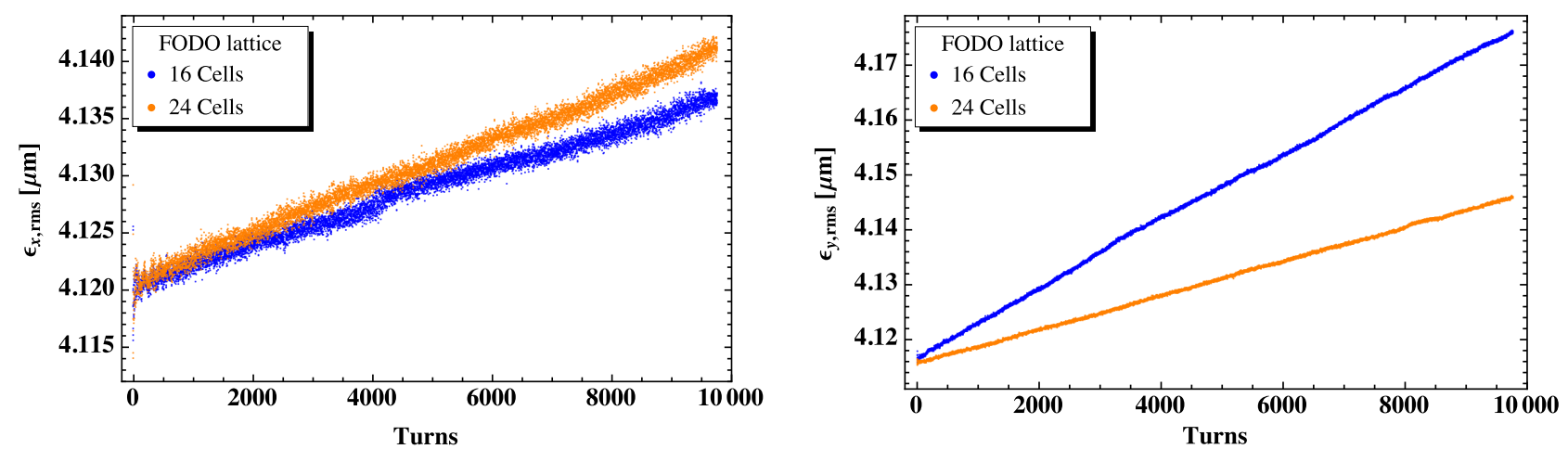

FIG. 18. Time evolution of horizontal (top) and vertical (bottom) rms emittance for the regular FODO lattices with 16 and 24 cells.

observed in the vertical plane for the 24-cell lattice with a smaller phase advance per cell compared to the 16-cell FODO lattice with a higher phase advance per cell. Nonetheless, it should be noted that the reduction of the emittance growth could also be due to the smaller variation of the beam size (Fig. 10) which is in this case mainly due to the decrease of the cell length with the number cells and not the phase advance per cell.

\section{F. Lattices with different dispersion suppression schemes}

Many modern high power hadron machines and also the proposed lattice for the RCS are based on a regular lattice with three to four inserted long straight sections in contrast to older machines like the PS Booster having a lattice built entirely out of equal cells. The straight sections in the modern lattices are usually required for injection, extraction, $\mathrm{rf}$ and eventually collimation and need to be dispersion free.

For the RCS two different dispersion suppression schemes were considered: (A) dispersion suppression by adjusting the phase advance to $2 \pi$ over the arc and with and without correction of the beta-beating with individual quadrupoles; and (B) half missing bend dispersion suppression, in which case the second dipole of the arc cell adjacent to the straight section is moved by half a cell into the straight section.

Both schemes are illustrated on the example of the 24cell FODO lattice and optics with $2 \times 2$ inserted straight sections shown in Fig. 20. Based on the lattices proposed for the RCS, the influence of these two dispersion suppression schemes on the time evolution of the emittance has been studied for three different lattices (Fig. 19): (1) the 24cell FODO lattice with $4 \times 1$ cell of straight section, (2) the 24-cell FODO lattice with $2 \times 2$ cells of straight section, and (3) the 21-cell FODO lattice with $3 \times 2$ cells of straight section.

For each lattice, the dispersion suppression schemes A and $\mathrm{B}$ have been compared and the simulation results for the different optics and lattices are summarized in Sec. IV F 2.

In general, scheme A without any individual quadrupoles is the most minimalistic and compact one as it allows one to build a lattice with only two quadrupole families and one family of dipoles, and thus does not require any space for a dedicated dispersion suppression section. The main disadvantage of this scheme is that the horizontal tune is directly tied to the number of cells per arc and the number of straight sections of the lattice, limiting the flexibility of the lattice and optics. In addition, the straight sections can induce large beta-beating and the dispersion suppression by
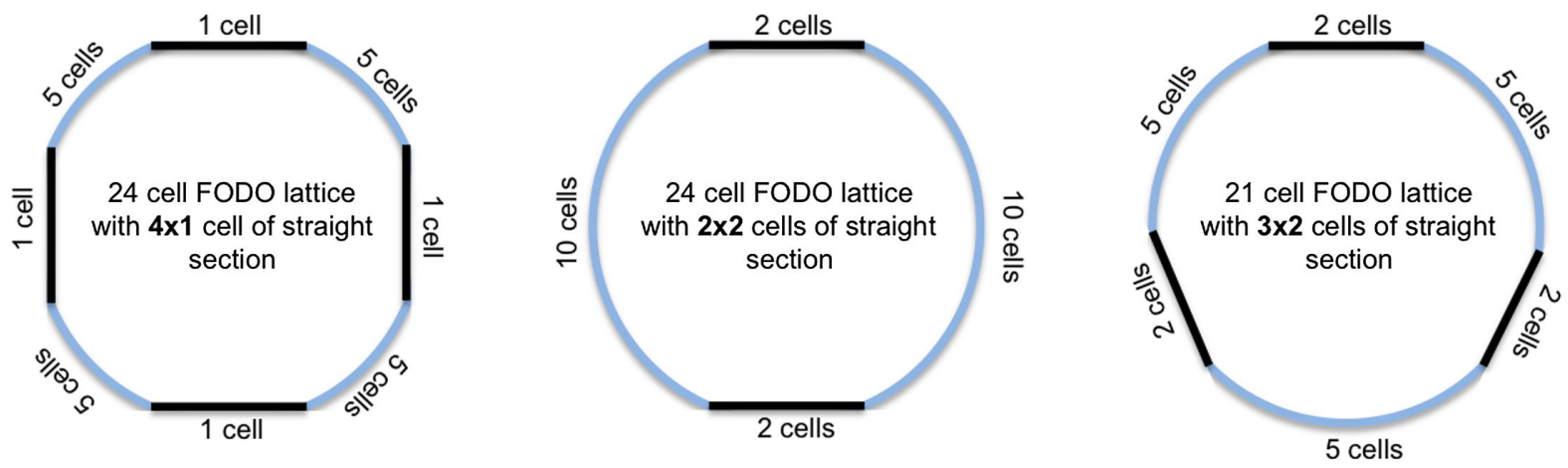

FIG. 19. Sketch of the 24-cell FODO lattice with $4 \times 1$ cells of straight sections and $2 \times 2$ cells of straight section, and the 21 -cell FODO lattice with $3 \times 2$ cells of straight section. 
adjusting the phase to $2 \pi$ over the arc in general leads to a large dispersion in the arc [Fig. 20 (top)]. The beta-beating can be corrected by allowing for individual quadrupoles next to the straight section with which the $\beta$-function can be matched to the arc. Regarding the dispersion suppression, no considerable reduction of the maximum dispersion could be achieved for the lattices studied with this method [Fig. 20 (middle)]. The consequences of a large dispersion in the arc will be described in detail in Sec. IV F 1. Compared to scheme A, the main advantage of the halfmissing bend dispersion suppression scheme (scheme B) is that it mitigates both the beta-beating and the large

$Q_{x} / Q_{y}=4.80 / 3.55$, no individual quadrupoles (no ind. quad.)
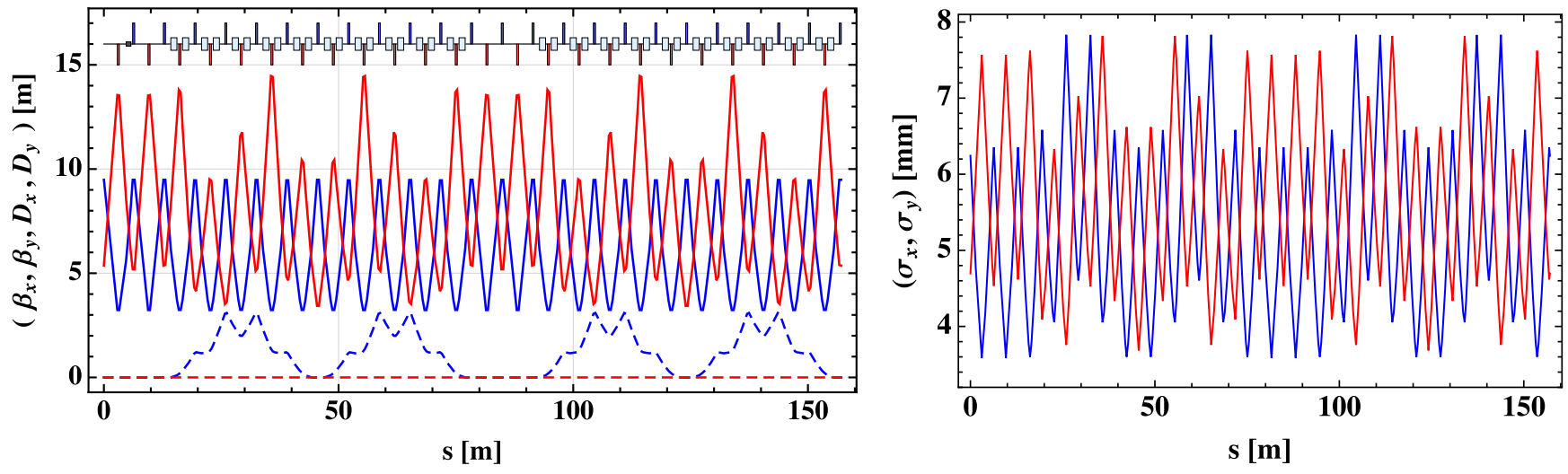

$Q_{x} / Q_{y}=4.38 / 3.55$, with individual quadrupoles (ind. quad.)
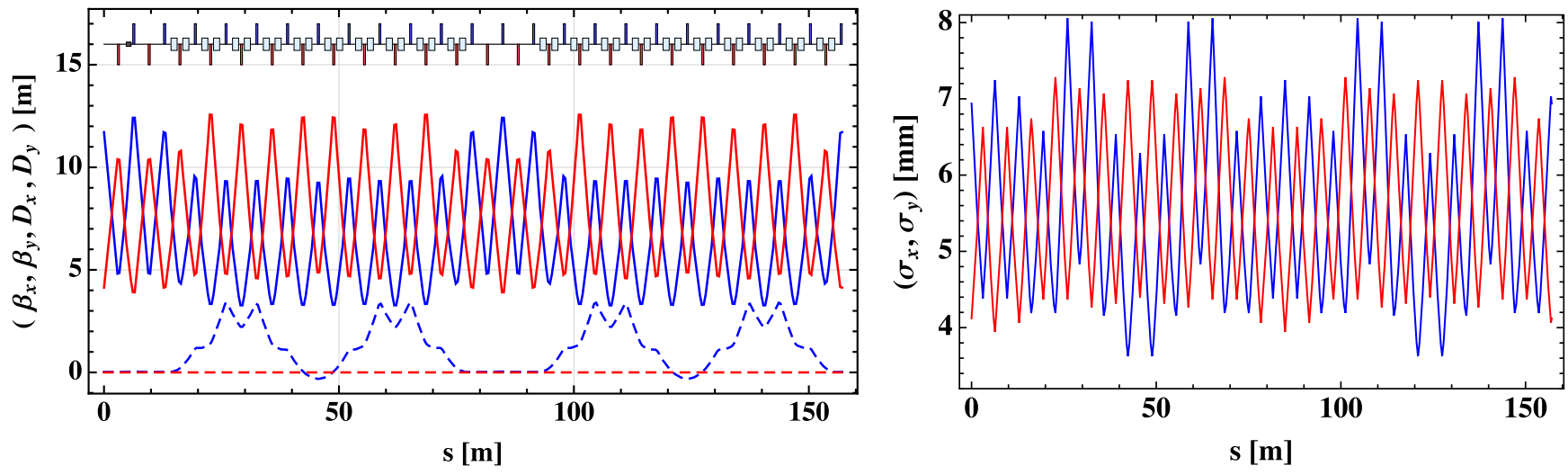

$Q_{x} / Q_{y}=4.38 / 3.55$, with half missing bend dispersion suppression (disp.)
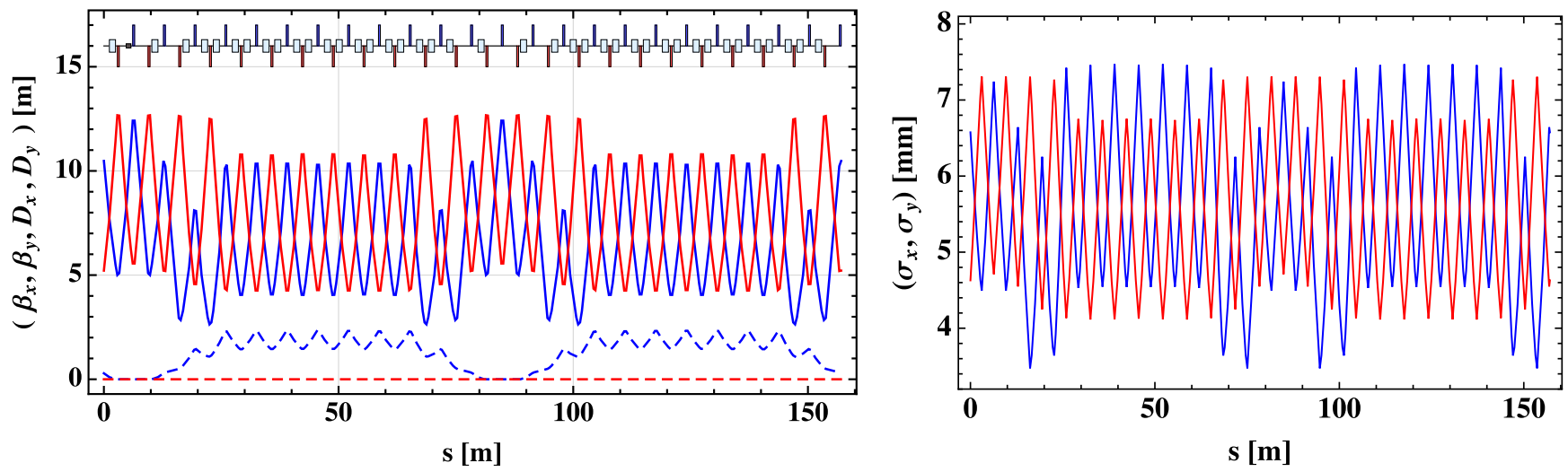

FIG. 20. Optics and one rms beam envelope of the 24-cell FODO lattice with $2 \times 2$ cells of straight section with only two quadrupole families (no ind. quad), with additional individual quadrupoles for beta-beating correction (ind. quad) and with a half missing bend dispersion suppression scheme (disp.). The beam envelope has been calculated using the design transverse normalized rms emittance of $2.5 \mu \mathrm{m}$ and the rms momentum spread corresponding to the design longitudinal emittance of $2.8 \mathrm{eVs}$. 
dispersion in the arc resulting in a much smoother beam envelope [Fig. 20 (bottom)]. On the other hand, valuable space is used for the dispersion suppressor, making the machine less compact than if scheme A was used.

\section{Influence of dispersion}

Analyzing the time evolution of the emittances of the lattices with inserted straight sections, an initial oscillation of the horizontal emittance during the first 40 turns is observed (Fig. 27), which is not visible in the case of any of the lattices without inserted straight sections. For illustration, the time evolution of the emittances only over the first 40 turns for the 24-cell FODO lattice with $4 \times 1$ cell of straight section and different working points is shown in Fig. 21. From Fig. 21 it also becomes apparent that the amplitude of this oscillation increases for working points closer to the integer resonance. This initial oscillation of the horizontal emittance can be explained by a dispersion and smaller betatron mismatch induced by space-charge. The dispersion and second order dispersion both satisfy differential equations similar to the one of a closed-orbit distortion due to dipole field errors, explicitly [20]:

$$
\begin{aligned}
D^{\prime \prime}+\left(k_{x}+\frac{1}{\rho^{2}}\right) D & =\frac{1}{\rho}, \\
D_{2}^{\prime \prime}+\left(k_{x}+\frac{1}{\rho^{2}}\right) D_{2} & =\kappa\left(\rho, \rho^{\prime}, k, k^{\prime}, D, D^{\prime}\right),
\end{aligned}
$$

where $\kappa\left(\rho, \rho^{\prime}, k, k^{\prime}, D, D^{\prime}\right)$ stands for a polynomial in $\rho, \rho^{\prime}, k, k^{\prime}, D$ and $D^{\prime}$, and are thus also most sensitive to tunes close to the integer resonance. Therefore, if the particle tune is moved closer to the integer resonance by the space-charge detuning or by directly moving the tune, the dispersion mismatch increases. This effect is illustrated in Fig. 22, which shows the dependence of the oscillation on the beam intensity and thus space-charge detuning. Consistently, the oscillation amplitude decreases with the beam intensity, in which case the space-charge detuning decreases, and vanishes completely for the case of zero intensity.

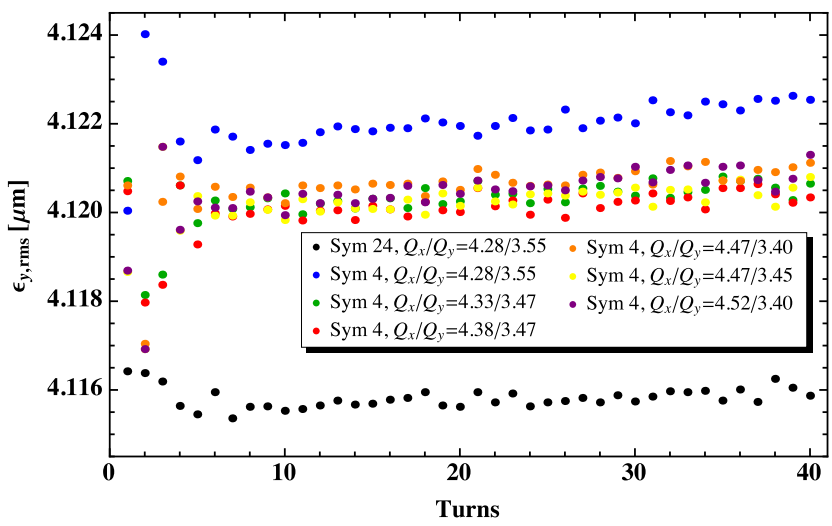

FIG. 21. Time evolution over the first 40 turns of the horizontal (left) and vertical (right) rms emittance for the regular 24-cell FODO lattice (Sym 24) and the 24-cell FODO lattice with $4 \times 1$ cells straight sections (Sym 4) for different working points. The initial oscillation of the horizontal emittance decreases if the working point is moved away from the integer resonance.
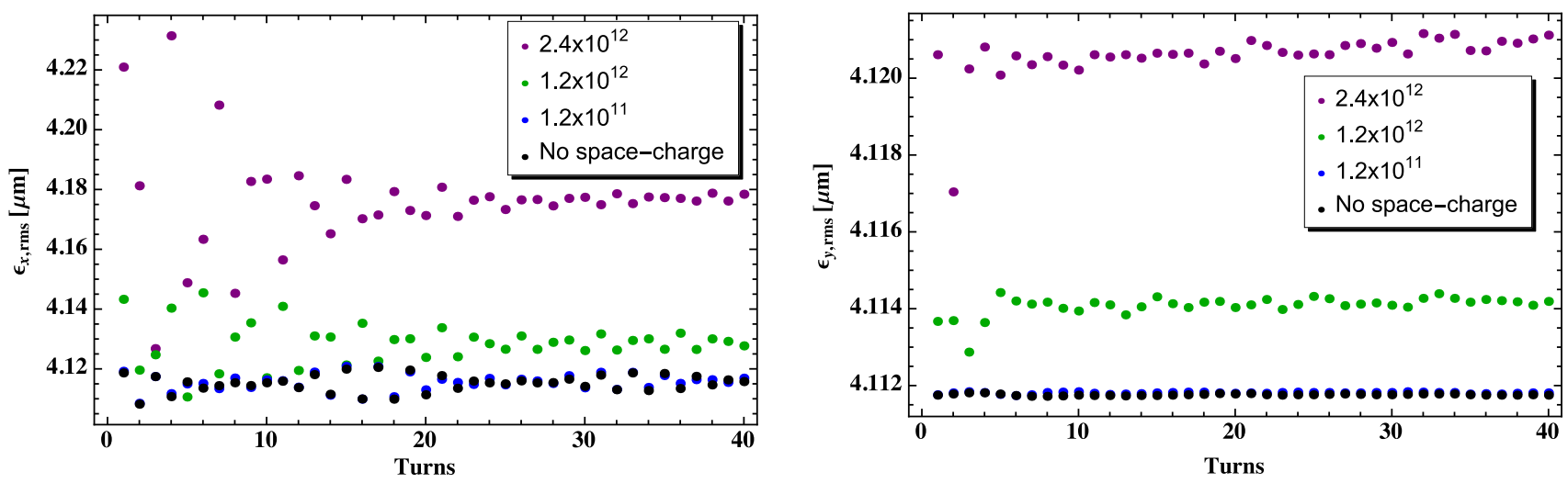

FIG. 22. Time evolution over the first 40 turns of the horizontal (left) and vertical (right) rms emittance for the 24-cell FODO lattice with $4 \times 1$ cell inserted straight sections with a working point of $Q_{x} / Q_{y}=4.47 / 3.45$. The initial oscillation of the horizontal emittance decreases with intensity due to the decreasing dispersion mismatch. 
It should be also mentioned that the emittance is calculated in PTC-ORBIT by first subtracting the closedorbit shift,

$$
x_{\mathrm{co}}=D_{x} \delta,
$$

from the horizontal position, where only the linear dispersion is taken into account, and then determining the emittance from the first and second beam moments. Therefore, if the dispersion becomes nonlinear, the emittance is then no longer correctly calculated. In the case of the lattices presented in this paper, the second-order dispersion of the lattice itself turned out to be also a measure for the nonlinearity of the dispersion under spacecharge. For this reason, the emittance calculated with PTCORBIT and also the emittance taking the second order dispersion into account are both shown in the following. The emittance taking the second order dispersion into account is obtained by subtracting the closed orbit,

$$
x_{\mathrm{co}}=D_{x} \delta+\frac{1}{2} D_{2 x} \delta^{2},
$$

from the horizontal position and then calculating the beam moments. The difference between the two values is then an indication for the nonlinearity of the dispersion.

In the case of the simulations presented in this paper, the initial mismatch between the optical parameters with spacecharge and the initial distribution matched to the optical parameters of the linear lattice without space-charge leads to filamentation of the mismatched distribution, and thus later to emittance growth. In a real machine, for which the injected beam distribution is usually matched to the linear optics without space-charge, a similar effect would be observed resulting in a large blow-up of the horizontal emittance at injection.

\section{Comparison of the dispersion suppression by adjusting the phase advance to $2 \pi$ over the arc (scheme A) and the half missing bend dispersion suppressor scheme (scheme B)}

In order to compare the two different dispersion suppressor schemes, the three aforementioned lattices have been studied: (1) 24-cell FODO lattice with $4 \times 1$ cells of straight section, (2) 24-cell FODO lattice with $2 \times 2$ cells of straight section, and (3) 21-cell FODO lattice with $3 \times 2$ cells of straight section.

In the case of the 24-cell lattice with $4 \times 1$ cells of straight section, it is not possible to construct a half missing bend dispersion suppressor (scheme B) and thus only scheme A has been studied for this lattice. The simulation results are summarized in Table XI and the time evolution of the emittance shown in Fig. 27.

For the 24-cell FODO lattice with $4 \times 1$ and $2 \times 2$ cells of straight section the condition for dispersion suppression of a phase advance of $n \times 2 \pi$ over the arc is fulfilled for a horizontal working point of $Q_{x}=4.8$. This working point is not advisable as it is located close to the systematic $5 Q_{x}$ resonance and limited on the lower end (towards the core of the bunch) by the $2 Q_{x}$ resonance, and a large emittance growth is indeed observed for the lattice without individual quadrupoles. For the lattices with individual quadrupoles the working point has therefore been changed in order to avoid the $5 Q_{x}$ and $2 Q_{x}$ resonance. In addition, in order to reduce the initial oscillation of the horizontal emittance, the horizontal working point has been increased compared to the lattices presented earlier in this paper-away from the integer resonance (Sec. IV F 1) - and the vertical working point slightly reduced. Compared to the lattice with only two quadrupole families, the emittance growth of the lattices with individual quadrupoles is small, but large in comparison to the regular 24-cell FODO lattice, in

TABLE XI. Deviation of the beam size $\Delta \sigma_{z}$ as defined in Eq. (13) and relative emittance growth for the 24-cell FODO lattice with and without straight sections and the 21-cell FODO lattice with and without straight sections. Scheme A without individual quadrupoles is abbreviated by no ind, scheme A with individual quadrupoles by no ind and scheme B with disp in agreement with the labeling

\begin{tabular}{|c|c|c|c|c|c|c|c|}
\hline Lattice & Disp scheme & $Q_{x} / Q_{y}$ & Symmetry & $\Delta \sigma_{x}[\mathrm{~mm}]$ & $\Delta \sigma_{y}[\mathrm{~mm}]$ & $\frac{\Delta \epsilon_{x}}{\epsilon_{x, 0}}\left[10^{-2}\right]$ & $\frac{\Delta \epsilon_{y}}{\epsilon_{y, 0}}\left[10^{-2}\right]$ \\
\hline 24 FODO, regular & & $4.28 / 3.55$ & 24 & 2.80 & 2.71 & 0.78 & 0.89 \\
\hline 24 FODO, $4 \times 1$ cell & No ind & $4.80 / 3.55$ & 4 & 4.24 & 3.85 & 11.03 & 9.60 \\
\hline 24 FODO, $4 \times 1$ cell & Ind & $4.47 / 3.45$ & 4 & 4.02 & 3.01 & 3.48 & 1.78 \\
\hline 24 FODO, $2 \times 2$ cell & No ind & $4.80 / 3.55$ & 2 & 4.25 & 4.13 & 66.29 & 22.00 \\
\hline 24 FODO, $2 \times 2$ cell & Ind & $4.38 / 3.55$ & 2 & 4.43 & 3.34 & 6.39 & 2.72 \\
\hline 24 FODO, $2 \times 2$ cell & Disp & $4.38 / 3.55$ & 2 & 4.00 & 3.19 & 2.01 & 1.92 \\
\hline 24 FODO, $2 \times 2$ cell & Disp & $4.28 / 3.55$ & 2 & 4.04 & 3.22 & 2.44 & 2.12 \\
\hline 21 FODO, regular & & $4.47 / 3.42$ & 21 & 3.50 & 3.15 & 0.35 & 0.96 \\
\hline 21 FODO, $3 \times 2$ cell & No ind & $4.47 / 3.42$ & 3 & 5.58 & 5.50 & 2.73 & 4.74 \\
\hline 21 FODO, $3 \times 2$ cell & Ind & $4.47 / 3.42$ & 3 & 6.04 & 3.84 & 3.84 & 5.06 \\
\hline 21 FODO, $3 \times 2$ cell & Disp match 1 & $4.47 / 3.42$ & 3 & 4.57 & 3.30 & 2.60 & 2.48 \\
\hline 21 FODO, $3 \times 2$ cell & Disp match 2 & $4.47 / 3.42$ & 3 & 4.25 & 3.98 & 4.45 & 3.80 \\
\hline 21 FODO, $3 \times 2$ cell & Disp & $4.45 / 3.42$ & 3 & 4.24 & 4.04 & 6.63 & 6.82 \\
\hline
\end{tabular}
in Fig. 27. 

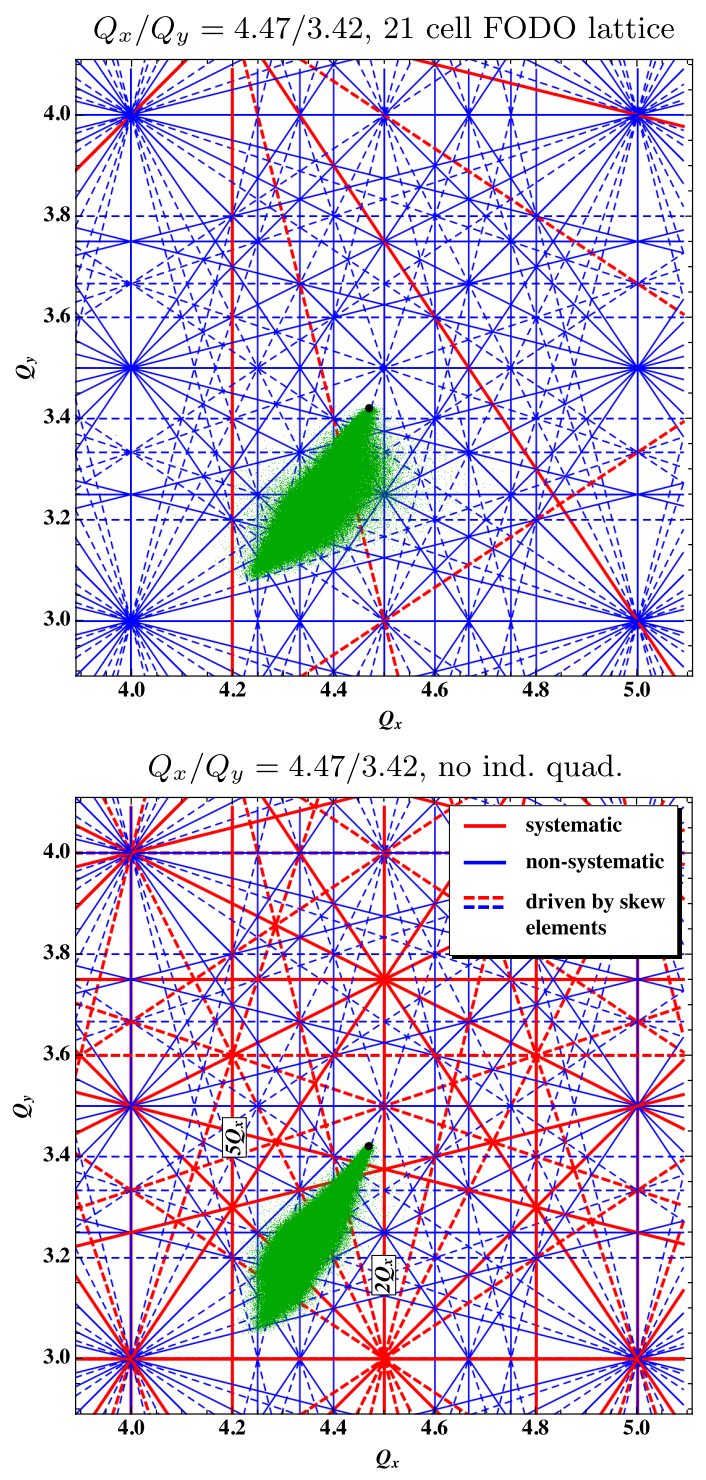

FIG. 23. Resonance diagram up to 5th order and tune footprint obtained with PTC-ORBIT of the initial distribution for the regular 21-cell FODO lattice (left) and the 21-cell FODO lattice with $3 \times 2$ cells of straight sections, dispersion suppression by adjusting the phase advance to $2 \times 2 \pi$ over the arc and only two quadrupole families. Both lattices are matched to the working point $Q_{x} / Q_{y}=4.47 / 3.42$. The initial distribution has been matched in both cases, taking only the linear dispersion into account.

particular in the horizontal plane for which the initial mismatch could not be avoided entirely. Particularly interesting is that in the case of the dispersion suppressor scheme B (only for $2 \times 2$ cells on straight section), the initial oscillation of the horizontal emittance vanishes entirely (Fig. 27). In addition, the overall emittance growth in the horizontal as well as in the vertical plane is slightly reduced.

Also in the case of the 21-cell FODO lattice with $3 \times 2$ cells of straight section, the horizontal working point fulfilling the condition for dispersion suppression of $2 \times 2 \pi$ phase advance over the arc is disadvantageous as it lies directly on the systematic $5 Q_{x}$ resonance, explicitly $Q_{x}=4.2$, and a large emittance growth is also observed for the lattices with this working point. In particular, also the regular 21-cell FODO lattice with a working point of $Q_{x} / Q_{y}=4.28 / 3.55$ exhibits a large emittance growth (Fig. 11). The emittance growth can be related to the systematic $5 Q_{x}$ resonance, as an emittance growth comparable to the lowest one of all FODO cell lattices studied in this paper, the 24-cell regular FODO lattice, can be achieved by changing the horizontal working point to $Q_{x}=4.47$. An additional slight reduction could be achieved by lowering also the vertical tune slightly, leading to $Q_{x} / Q_{y}=4.47 / 3.42$, to which all 21-cell lattices with or without straight sections have been matched, except evidently the one without individual quadrupoles. In contrast to the 24-cell lattices with straight sections all 21-cell lattices do not show the initial oscillation of the horizontal emittance. Also the calculation of the emittance taking only the linear dispersion into account agrees well with calculation taking also the second order dispersion into account. This behavior suggests that the higher order dispersion effects have become less relevant or even negligible as the particles are trapped between the systematic $5 Q_{x}$ and $2 Q_{x}$ resonance visible in the tune footprint shown in Fig. 23. The proximity of the two systematic resonance makes the system sensitive to any small changes of the working point and also changes of the optics while keeping the working point constant. An example for the sensitivity to changes in the optics and working point are the different time evolution of the emittances for the three different optics of the dispersion suppression scheme B, where disp match 1 and disp match 2 in Fig. 27 are two different optics for the same lattice and working point of $Q_{x} / Q_{y}=4.47 / 3.42$ and $Q_{x} / Q_{y}=4.45 / 3.42$ disp an optics with a slightly different working point.

\section{Conclusion}

Comparing the two different dispersion suppression schemes, scheme A (dispersion suppression by adjusting the phase advance to $2 \pi$ over the arc) in general exhibits a large beta-beating if no additional individual quadrupoles are used for the beta-beating correction, and a large dispersion in the arc even with additional individual quadrupoles. Both effects lead to a stronger variation of the beam size. With scheme B in particular the dispersion in the arc can be considerably reduced leading to an overall smaller variation of the beam size compared to scheme A.

The above examples showed that for equal working points a larger variation of the beam size leads to a larger emittance growth if comparing the different dispersion suppression schemes. As a measure for the variation of the beam size, the difference between the maximum and minimum beam size, 


$$
\Delta \sigma_{z}=\Delta \sigma_{z, \max }-\Delta \sigma_{z, \min }, \quad z=x, y,
$$

can be employed. This criterion is better suited for the lattices with dispersion suppression than Eq. (9) as the symmetry of the lattice is kept in the case of the different dispersion suppression schemes. To summarize the results, the dependence of the maximum emittance growth on the variation of the horizontal and vertical beam size is shown in Fig. 24. Except for the 21-cell FODO lattices, a clear dependence of the emittance growth on the variation of the beam size is visible. In the case of the 21-cell FODO lattices with straight sections the particles are trapped between the $5 Q_{x}$ and $2 Q_{x}$ resonance making the lattices sensitive to any small changes in working point and optics.
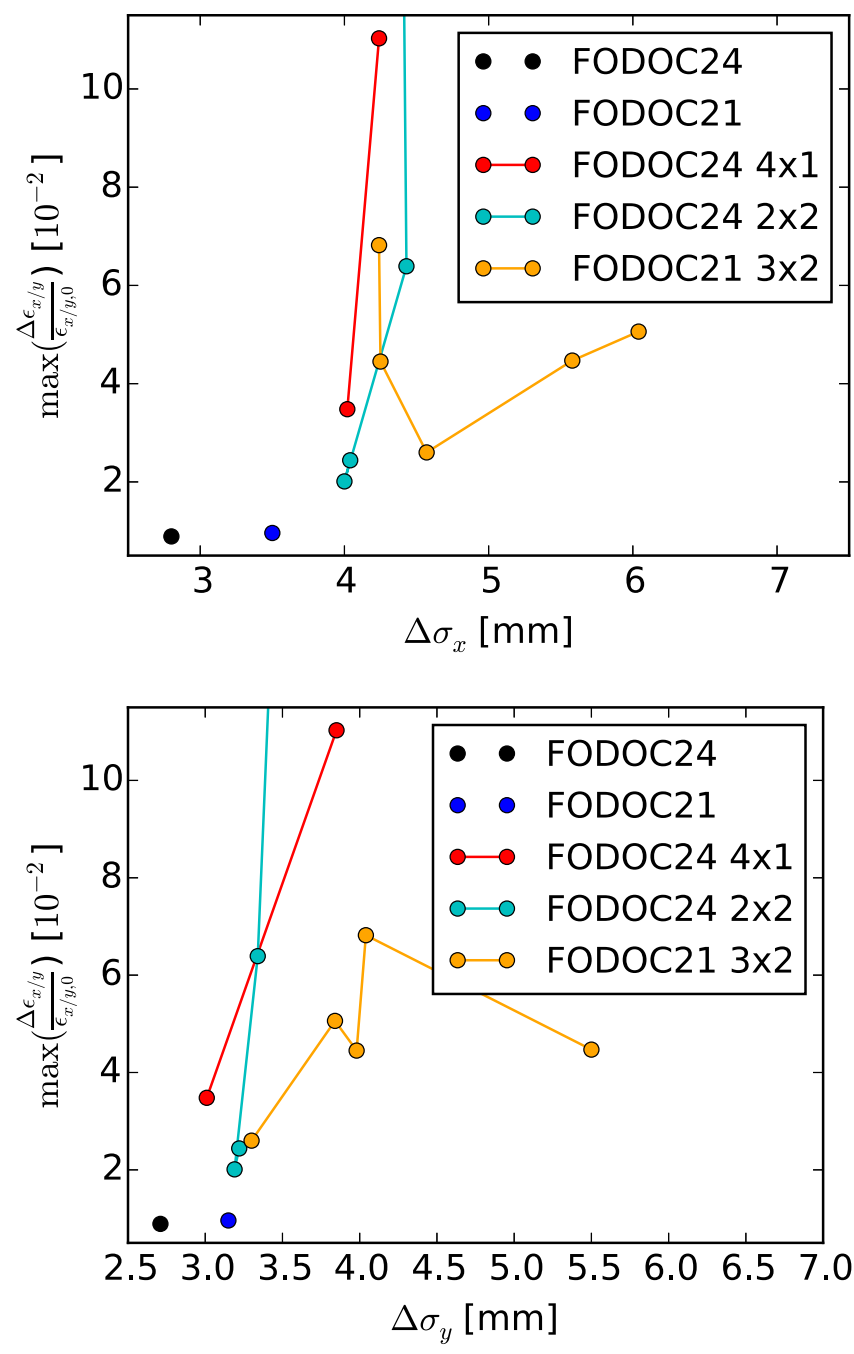

FIG. 24. Dependence of the maximum emittance growth on the variation of the beam size defined by Eq. (13) for the 24 and 21-cell FODO lattices with different dispersion suppressor schemes listed in Table XI.

\section{CONCLUSIONS}

The main results of the studies of different lattice types can be summarized in a list of lattice and optics characteristics and their influence on the time evolution of the rms emittance under consideration of direct space-charge effects. These lattice and optics characteristics are as follows.

Symmetry: In all cases, the crossing of systematic resonances led to an increase of the rms emittance growth. As the lattice symmetry defines which resonances are systematic and which ones are nonsystematic, the symmetry becomes a quite important characteristic of the lattice and optics.

The symmetry of a lattice can in general be broken by introducing a deviation of the beam size from the regular pattern. In case of the lattices studied, already a weak perturbation of the symmetry lead to an increased emittance growth. Furthermore, for only small perturbations, the lattice symmetry was partly preserved. In the case of large perturbations, no residual effect of the original lattice symmetry is visible (the lattice symmetry is reduced to one) and all lattices showed a large emittance growth.

Variation of the beam size: The original assumption was that a larger (but regular) variation of the beam size also results in a larger emittance growth. The only clear example for the influence of the variation of the beam size is the comparison of different cell types. In this case a larger emittance growth was indeed observed for the cells with larger variations of the beam size.

Dispersion suppressor scheme: Two dispersion suppressor schemes have been studied: (A) dispersion suppression by adjusting the phase advance to $2 \pi$ over the arc with and without correction of the beta-beating with individual quadrupoles; and (B) half missing bend dispersion suppressor, for which the second dipole of the arc cell adjacent to the straight section is moved by half a cell into the straight section.

An obvious disadvantage of lattices with a dispersion suppression by adjusting the phase advance to $n \cdot 2 \pi$ over the arc is the fixed working point in the case of the lattices with only two quadrupole families, and in addition the large beta-beating. By introducing more quadrupole families, both problems can be mitigated. All lattices with a dispersion suppression by adjusting the phase advance to $n \cdot 2 \pi$ over the arc show initial oscillations and increase of the horizontal emittance, in particular for working points close to the integer resonance. This attributes to an initial dispersion and betatron mismatch introduced by spacecharge, which increases for particles with a single particle tune approaching the integer resonance. This mismatch is especially strong for the dispersion suppression by adjusting the phase advance to $n \cdot 2 \pi$ over the arc, compared to the half missing bend dispersion suppression, due to the large maximum dispersion characteristic for this scheme.

In summary, the lattices with a half missing bend scheme all feature a flexible choice of the working point, a smaller 
variation of the beam size due to the regular dispersion in the arc, smaller beta-beating in the complete ring and, most importantly, a significant reduction of the initial oscillation of the horizontal emittance and of the overall emittance growth.

\section{ACKNOWLEDGMENTS}

We would like to thank several colleagues for input and helpful discussions: G. Arduini, H. Bartosik, B. Holzer, F. Schmidt and H. Schönauer.

\section{APPENDIX A: TUNE FOOTPRINTS AND RESONANCE DIAGRAMS}

For illustration the tune footprints and resonance diagrams are shown for the 16-cell triplet lattice with different symmetries in Fig. 25 and for the regular FODO cell lattice with different periodicities in Fig. 26.
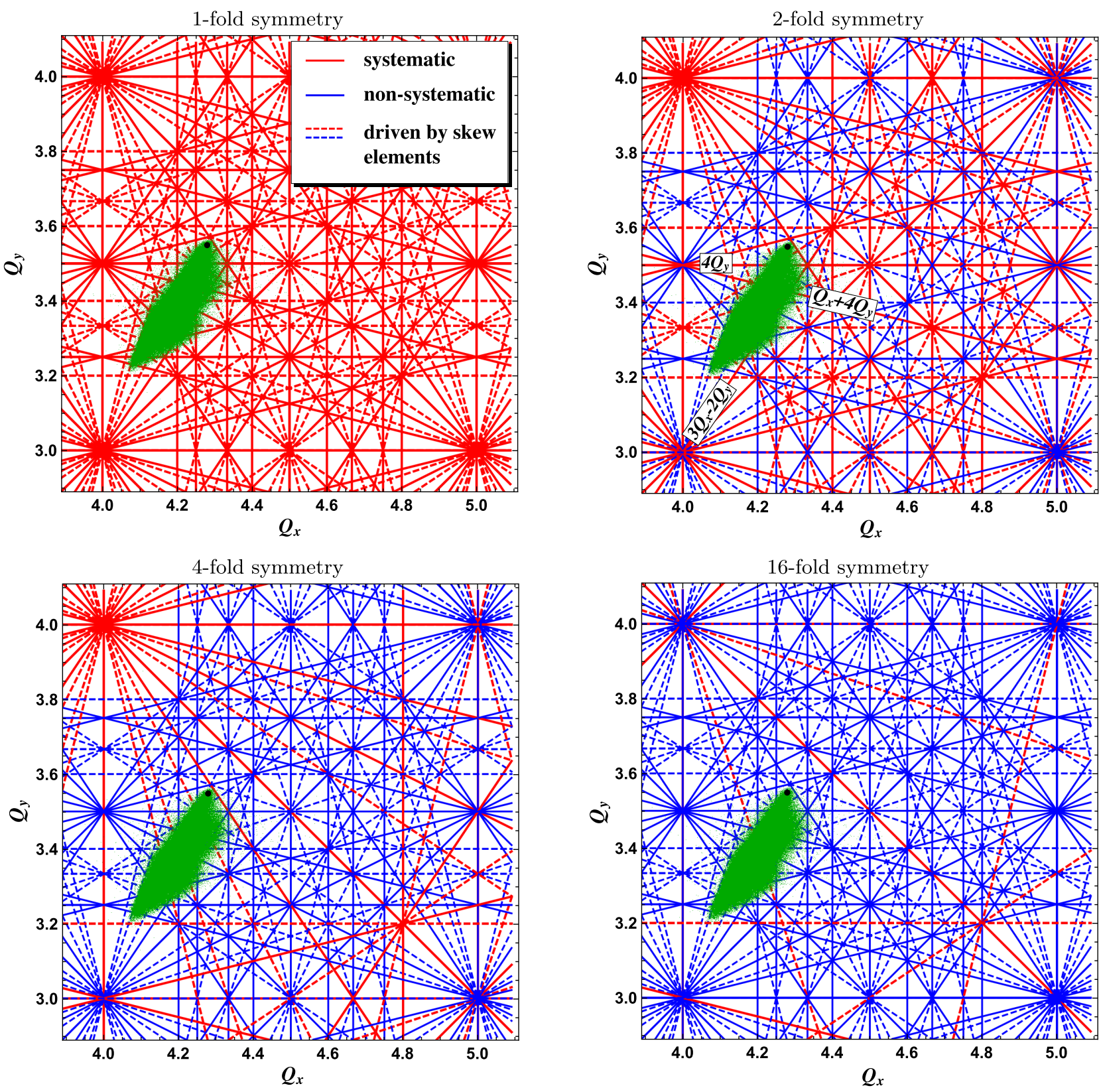

FIG. 25. Resonance diagram up to 5th order and tune footprint after 5000 turns for the 16-cell triplet lattice with onefold, twofold, fourfold and 16-fold symmetry. 

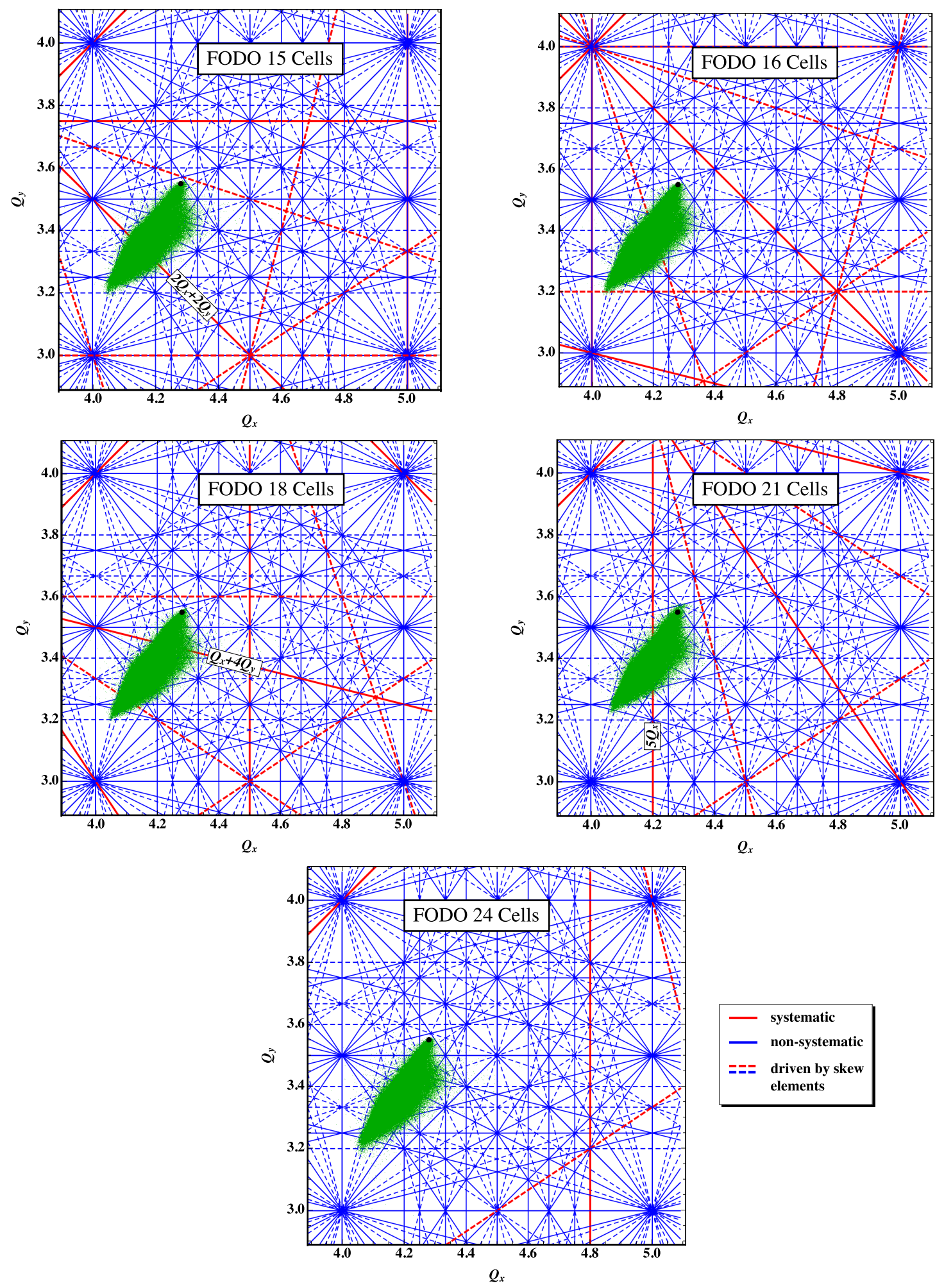

FIG. 26. Resonance diagram up to 5th order and tune footprint after 5000 turns for the regular FODO lattice with periodicity 15, 16, 18,21 , and 24. 


\section{APPENDIX B: TIME EVOLUTION OF THE EMITTANCE FOR LATTICES WITH DISPERSION SUPPRESSOR}

In Fig. 27 the time evolution of the emittance is shown for the 24 and 21 cell FODO lattices and different dispersion suppressor schemes in comparison to the regular 24 and 21 cell FODO lattice, which are discussed in further detail in Sec. IV F 1.
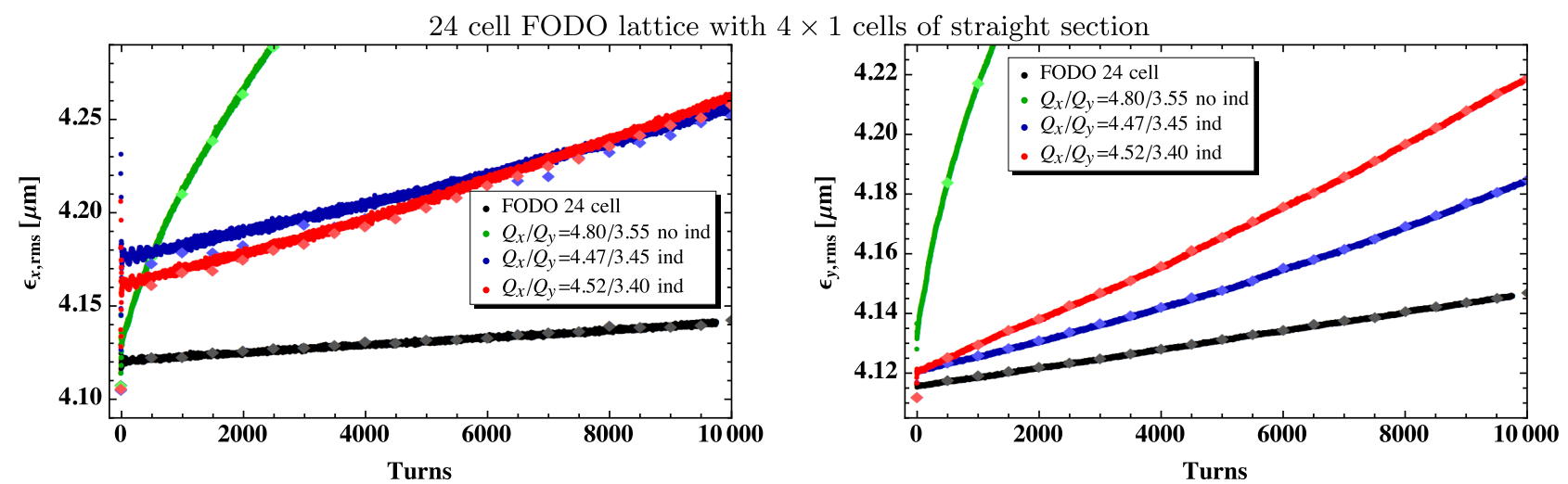

24 cell FODO lattice with $2 \times 2$ cells of straight section
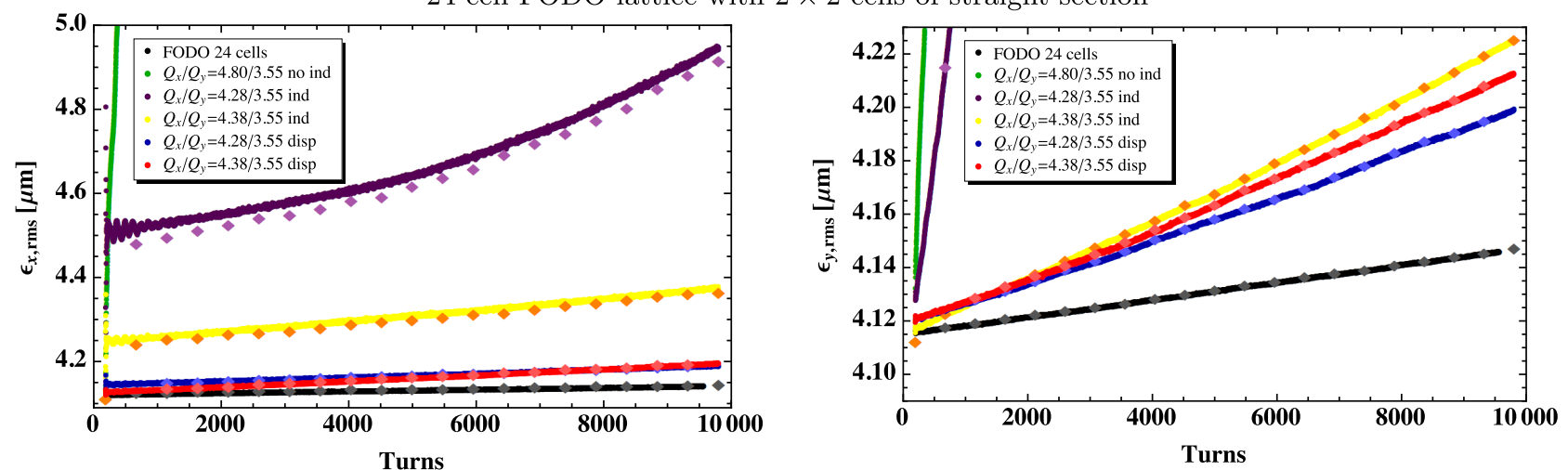

21 cell FODO lattice with $3 \times 2$ cells of straight section
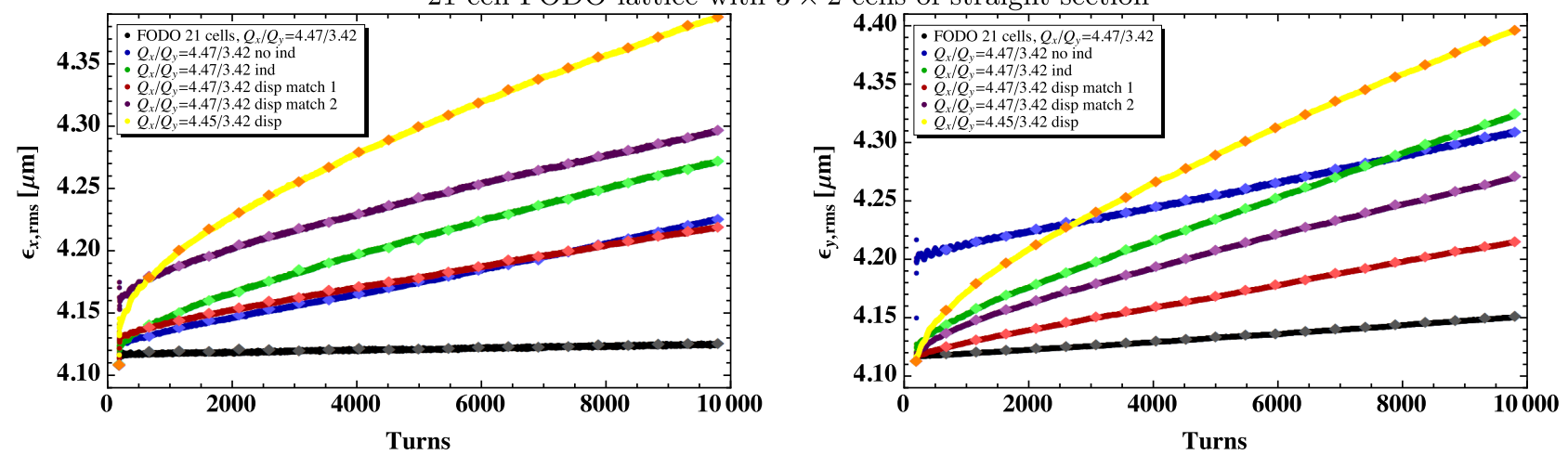

FIG. 27. Time evolution of horizontal (left) and vertical (right) rms emittance for the 24-cell FODO lattice with $4 \times 1$ (top), 24 -cell FODO lattices with $2 \times 2$ cells (center) and 21 -cell FODO lattices with $3 \times 2$ cells of straight section (bottom) with only two quadrupole families (no ind), with additional individual quadrupoles for beta-beating correction (ind) and with a half missing bend dispersion suppression scheme (disp), the latter only for 24-cell FODO lattices with $2 \times 2$ cells and 21-cell FODO lattices with $3 \times 2$ cells of straight section. For comparison also the time evolution of the emittance of the regular 24- and 21-cell FODO lattice is shown (FODO 24 cells and FODO 21 cells). The values indicated with diamonds are the emittances calculated including the second order dispersion. For all simulations the initial distribution has been created taking only the linear dispersion into account. 
[1] M. Giovannozzi et al., Possible improvements to the existing pre-injector complex in the framework of continued consolidation, in Proceedings of the Chamonix 2010 workshop on LHC performance (CERN, Geneva, Switzerland, 2010).

[2] K. Hanke et al., Study of a rapid cycling synchrotron to replace the CERN PS Booster, in Proceedings of the 2nd International Particle Accelerator Conference, San Sebastián, Spain, 2011 (2011).

[3] K. Hanke et al., PS Booster energy upgrade, feasibility study, first report, Technical Report, CERN, 2010, https://edms.cern.ch/document/1082646/3.

[4] B. Goddard et al., Can the proton injectors meet the HLLHC requirements after LS2?, in Proceedings of Chamonix 2011 workshop on LHC performance (CERN, Geneva, Switzerland, 2012).

[5] L. Rossi, LHC upgrade plans: Options and strategy, in Proceedings of the 2nd International Particle Accelerator Conference, San Sebastián, Spain, 2011 (2011).

[6] K. Hanke et al., Study of an energy upgrade of the CERN PS Booster, in Proceedings of the 2nd International Particle Accelerator Conference, San Sebastián, Spain, 2011 (2011).

[7] M. Fitterer, R. Garoby, M. Benedikt, H. Burkhardt, C. Carli et al., Lattice design of a RCS as possible alternative to the PS Booster upgrade, in Proceedings of the 2nd International Particle Accelerator Conference, San Sebastián, Spain, 2011 (2011).

[8] K. Shigaki, F. Noda, K. Yamamoto, S. Machida, A. Molodozhentsev et al., The JKJ lattice, AIP Conf. Proc. 642, 140 (2002).

[9] A. Lachaize, RCS design, Proc. Sci., Nufact08 (2008).
[10] S. Wang, Beam Dynamics Design of CSNS/RCS, ICFA Beam Dynamics Newsletter 40, 52 (2006).

[11] C. Carli, M. Benedikt, H. Damerau, R. Garoby, B. Goddard, K. Hanke, S. Hancock, and S. Gilardoni, Alternative/complementary possiblities, in Proceedings of Chamonix 2011 Workshop on LHC Performance (CERN, Geneva, Switzerland, 2011).

[12] K. Hanke et al., Feasibility study of a rapid cycling synchrotron to replace the PS Booster, Technical Report, CERN, 2011, https://edms.cern.ch/document/1154705/1.0.

[13] M. Fitterer, Design study of the large hadron electron collider and a rapid cycling synchrotron as alternative to the PS Booster upgrade at CERN, Report No. CERNTHESIS-2013-043.

[14] M. Benedikt, P. Collier, V. Mertens, J. Poole, and K. Schindl, LHC design report, CERN, Geneva, 2004.

[15] E. Forest, A. Molodojentsev, A. Shishlo, and J. Holmes, Synopsis of the PTC and ORBIT integration, KEK Internal Report No. 2007-4 (A), 2007.

[16] E. Forest, F. Schmidt, and E. McIntosh, Introduction to the polymorphic tracking code, KEK Report, 2002.

[17] J. D. Galambos, J. A. Holmes, and D. K. Olsen, ORBIT user manual, NS/ORNL/AP technical note, 1999.

[18] O. Boine-Frankenheim, I. Hofmann, J. Struckmeier, and S. Appel, Artificial collisions, entropy and emittance growth in computer simulations of intense beams, Nucl. Instrum. Methods Phys. Res., Sect. A 770, 164 (2015).

[19] R. Baartman, Betatron resonances with space charge, AIP Conf. Proc. 448, 56 (1998).

[20] A. Verdier, Chromaticity, CERN Accelerator School (1995), pp. 77-100. 